\title{
THE ROOTS OF TRIGONOMETRIC INTEGRALS
}

\author{
By N. G. De BruiJN
}

1. Introduction. Concerning the roots of trigonometric integrals G. Pólya (see references at the end of the paper) has proved a number of results which he derived from properties of the roots of polynomials. He proved, for instance, the reality of all the roots of the following functions:

$$
\begin{array}{lr}
\int_{-\infty}^{\infty} e^{-t^{2 n} e^{i z t} d t} & (n=1,2,3, \cdots) ; \\
\int_{-\infty}^{\infty} C(t) e^{i z t} d t & (\lambda>0),
\end{array}
$$

where $C(t)=\exp (-\lambda \cosh t)$, and

$$
\int_{-\infty}^{\infty} \exp \left(-a t^{4 n}+b t^{2 n}+c t^{2}\right) \exp i z t d t
$$

where $a>0, b$ real, $c \geq 0, n=1,2,3, \cdots$. (See concerning (1.1), [7], [8]; concerning (1.2), [6], [8]; concerning (1.3), [8].)

Another important result of Pólya is the following one (see [8]): Suppose that the function $F(t)$ of the real variable $t$ satisfies

$$
F(t) \text { integrable over }-\infty<t<\infty ; F(t)=(F(-t))^{*},-\infty<t<\infty ;
$$

$$
F(t)=O\left(e^{-|t|^{b}}\right) \text { for } t \rightarrow \pm \infty, b>2 .
$$

(The ${ }^{*}$ indicates the conjugate imaginary.)

Let $\varphi(t)$ be an integral function of genus 0 or 1 , with real roots only, and let the number $\gamma$ be $\geq 0$. If the function $F(t)$ is such that all the roots of the integral

$$
\int_{-\infty}^{\infty} F(t) e^{i z t} d t
$$

are real, then the same holds for the function $\int_{-\infty}^{\infty} F(t) \varphi(i t) e^{\gamma t^{2}} e^{i z t} d t$.

The function $\varphi(i t) e^{\gamma t^{2}}$ is easily seen to be the limit of a sequence of polynomials, all of whose roots are purely imaginary. Pólya's result, stated in other words, is that these functions are universal factors, which conserve the reality of the roots of any trigonometric integral whose integrand satisfies (1.4). Pólya also proved that the functions $\varphi(i t) e^{\gamma t^{2}}$ indicated above are the only analytical functions with this property. The latter result will not be used in the present paper.

Received July 16, 1948. 
In the sequel we continue Polya's researches. Our main results are

THEOREM 1. Let $f(t)$ be an integral function of $t$ and such that its derivative $f^{\prime}(t)$ is the limit (uniformly in any bounded domain of the t-plane) of a sequence of polynomials, all of whose roots lie on the imaginary axis. Suppose furthermore that $f(t)$ is not a constant, and that $f(t)=f(-t), f(t) \geq 0$ for real values of $t$. Then the integral $\int_{-\infty}^{\infty} e^{-f(t)} e^{i z t} d t$ has real roots only.

(The conditions $(f(t)=f(-t), f(t) \geq 0)$ may be replaced by weaker ones, namely, " $f(t)=(f(-t))^{*}, \operatorname{Re} f(t) \geq 0$ for $-\infty<t<\infty$ ", if $f(z)$ is a polynomial or a function of the type (1.6) (see Theorems 19 and 20 respectively). It is not easy to see whether the latter set of conditions is sufficient in the general case.)

Pólya's results (1.1) and (1.2) are special cases of this one, but (1.3) is not.

Theorem 2. Let $N$ be a positive integer and put

$$
P(t)=\sum_{-N}^{N} p_{n} e^{n t} \quad\left(\operatorname{Re} p_{N}>0 ; p_{n}^{*}=p_{-n}, n=0,1,2, \cdots\right) .
$$

Let the function $q(x)$ be regular in the sector $-\pi / 2 N-N^{-1} \arg p_{N}<\arg x<$ $\pi / 2 N-N^{-1}$ arg $p_{N}$ and on its boundary, with possible exception of $x=0$ and $x=\infty$ which may be poles (of arbitrary finite order) for $q(x)$. Furthermore suppose

$$
(q(x))^{*}=q\left(1 / x^{*}\right)
$$

in this sector (in other words, $q(x)$ is real for $|x|=1$. Then all but a finite number of roots of the function

$$
\Phi(z)=\int_{-\infty}^{\infty} e^{-P(t)} Q(t) e^{i z t} d t \quad\left(Q(t)=q\left(e^{t}\right)\right)
$$

are real.

It may be remarked that our method fails to give any useful information concerning the number and location of the non-real roots of (1.8) in the general case, so that this very peculiar result may be of very little practical importance.

The special functions $(C(t)=\exp (-\lambda \cosh t))$

$$
\Psi(z)=\int_{-\infty}^{\infty} C(t) \sum_{-N}^{N} \alpha_{n} e^{n t} e^{i z t} d t \quad\left(\lambda>0, \alpha_{n}^{*}=\alpha_{-n}\right),
$$

which have $N$ pairs of non-real roots at most (Theorem 21), may be of some interest since the Riemann $\xi$-function can be approximated by functions of this type (see [8]). It will be worthwhile to determine classes of functions of this type with the property that all the roots are real. We shall study these questions in $\$ 6$.

(Those readers who are mainly interested in considerations concerning the $\xi$-function may omit the proof of Theorem 1 and the related results in $\$ 5$, and in Theorem 2 need to consider the case $P(t)=\lambda\left(e^{t}+e^{-t}\right)$ only. In that case the complicated $\$ 4$ is superfluous since the results of that section then reduce to well-known asymptotic formulas concerning the $\Gamma$-function.) 
In $\$ 7$ we expose what progress has been made in this paper in the direction of the Riemann hypothesis, and also how small this progress is.

An outline of the proofs of Theorems 1 and 2 concludes our introduction.

Sections 2 and 3 will furnish functions $S(t)$ which are special universal factors in Pólya's sense but which have stronger properties than those stated above. A function $S(t)$ of the real variable $t$, satisfying $S(t)=(S(-t))^{*}$ will be called a strong universal factor if it joins properties $(\alpha)$ and $(\beta)$ below, for any function $F(t)$ satisfying (1.4).

( $\alpha$ ) If the roots of (1.5) lie in a strip $|\operatorname{Im} z| \leq \Delta(\Delta>0)$, then those of $\int_{-\infty}^{\infty} F(t) S(t) e^{i z t} d t$ lie in a strip $|\operatorname{Im} z|<\Delta_{1}$, where $\Delta_{1}<\Delta, \Delta_{1}$ independent of $F(t)$.

( $\beta)$ If $F(t)$ is such that, for any $\epsilon>0$, all but a finite number of roots of (1.5) lie in the strip $|\operatorname{Im} z| \leq \epsilon$, then the function $\int_{-\infty}^{\infty} F(t) S(t) e^{i z t} d t$ has only a finite number of non-real roots.

It will be evident from $(\alpha)$ that any strong universal factor is a universal factor in Pólya's sense.

A function $S(t)$ of the type

$$
S(t)=\sum_{-N}^{N} a_{n} e^{n \lambda t} \quad\left(\lambda>0, a_{n}=a_{-n}^{*}\right)
$$

is a strong universal factor if all its roots lie on the imaginary axis. (Conversely, if $S(t)$ is a universal factor and if it is of the type (1.10), then its roots lie on the imaginary axis. This follows from Pólya's result on universal factors.) This result is obtained by generalizing a theorem of J. L. W. V. Jensen on the location of the roots of the derivative of a polynomial with real coefficients (\$2) and applying it to integral functions (\$3).

The functions $e^{\gamma t^{2}}, \gamma>0$, also turn out to have property $(\alpha)$, but it is doubtful whether they have property $(\beta)$.

The functions (1.8) will be shown to have but a finite number of roots outside any strip $|\operatorname{Im} z| \leq \epsilon, \epsilon>0$. This will be carried out by proving asymptotic formulas for $\Phi(z)$, depending on the expansion (5.7). In that formula an auxiliary function $H(s)$ occurs which is a generalization of the $\Gamma$-function. Asymptotic formulas for $H(s)$ will be derived in $\$ 4$.

Now let $P(t)$ and $Q(t)$ satisfy the conditions of Theorem 2 ; then also $P(t)$ and $Q(t) /\left(e^{t}+2+e^{-t}\right)$ satisfy these conditions. From what is said above it is evident that the function $\int_{-\infty}^{\infty} e^{-P(t)} Q(t)\left(e^{t}+2+e^{-t}\right)^{-1} e^{i x t} d t$ has but a finite number of roots outside any strip $|\operatorname{Im} z| \leq \epsilon$. Now applying property $(\beta)$ with $S(t)=e^{t}+2+e^{-t}$ we obtain Theorem 2 .

In order to sketch the proof of Theorem 1, let $P(t)$ satisfy the conditions of Theorem 2 and suppose that $P^{\prime}(t)$ has purely imaginary roots only. Let $\Delta$ be the smallest number with the property that the roots of $\Phi_{1}(z)=\int_{-\infty}^{\infty} e^{-P(t)} e^{i z t} d t$, which has but a finite number of non-real roots by virtue of Theorem 2, lie in the strip $|\operatorname{Im} z| \leq \Delta$, and suppose $\Delta>0$. The function $P^{\prime}(t)$ is a strong universal factor and hence, by $(\alpha)$, the roots of $\int_{-\infty}^{\infty} e^{-P(t)} i P^{\prime}(t) e^{i z t} d t$ lie in a strip 
$|\operatorname{Im} z| \leq \Delta_{1}, \Delta_{1}<\Delta$. But it is easily seen from partial integration that the latter integral equals $-z \Phi_{1}(z)$. It follows that the roots of $\Phi_{1}(z)$ also lie in the strip $|\operatorname{Im} z| \leq \Delta_{1}$. This contradicts the minimum property of $\Delta$. Hence $\Delta=0$ and all the roots of $\Phi_{1}(z)$ are real.

It will be relatively easy to extend this to the integrals of Theorem 1 on considering $F(t)$ as the limit of a sequence of functions $P(t)$.

The following notations are used throughout the paper.

$\operatorname{Re} \alpha$ and $\operatorname{Im} \alpha$ denote the real and imaginary parts of $\alpha: \alpha=\operatorname{Re} \alpha+i \operatorname{Im} \alpha ; \alpha^{*}$ denotes the conjugate of $\alpha$. If $f(z)$ is a function of the complex variable $z$, then $f^{*}(z)$ is defined by $f^{*}(z)=\left(f\left(z^{*}\right)\right)^{*}$. A polynomial or integral function $f(z)$ is called real if $f(z) \equiv f^{*}(z)$, that is to say if $f(z)$ is real for real values of $z$.

All the trigonometric integrals considered in this paper are real integral functions of $z$.

2. Theorems on polynomials. We shall deal with linear combinations of the type (2.3) for a given polynomial $f(z)$ with real coefficients; the simplest case is $f_{1}(z)=f(z+i)+f(z-i)$. Several properties of the roots of $f_{1}(z)$ are known; they all express in some way that the roots of $f_{1}(z)$ lie closer to the real axis than those of $f(z)$.

1. The number of non-real roots of $f_{1}(z)$ does not exceed that of $f(z)$. (This is a special case of Poulain's theorem. See [11; Abschn. VI, Aufg. 63].)

2. If the roots of $f(z)$ lie in the strip $|\operatorname{Im} z| \leq 1$, then $f_{1}(z)$ has real roots only. Namely, $|f(z+i)| \neq|f(z-i)|$ for $\operatorname{Im} z \neq 0$. (Properties 1 and 2 are contained as special cases in Theorem 9a.)

3. If $\alpha_{1}, \cdots, \alpha_{n}$ are the roots of $f(z)$ and $\beta_{1}, \cdots, \beta_{n}$ those of $f_{1}(z)$, then $\sum_{1}^{n}\left|\operatorname{Im} \beta_{\nu}\right| \leq \sum_{1}^{n}\left|\operatorname{Im} \alpha_{v}\right|$. (See [1; Theroem 5].)

These properties are meant for illustration and will not be used in the present paper. We shall now derive a new result of this type, Theorem 3, which forms the base of our paper. It is a generalization of the second property above. We first prove

Lemma 1. Put $z=x+i y$ ( $x$ and $y$ real), and $f(z)=z^{2}+\Delta^{2}$ where $\Delta \geq 0$; let $\lambda$ be a positive number. If $\mu$ is defined by $\mu=\left(\Delta^{2}-\lambda^{2}\right)^{\frac{1}{2}}(\Delta>\lambda)$ and $\mu=0$ $(\Delta \leq \lambda)$, then we have $|f(z+i \lambda)|>|f(z-i \lambda)|$ if $\left(|z|^{2}-\mu^{2}\right) y>0$ and $|f(z+i \lambda)|<|f(z-i \lambda)|$ if $\left(|z|^{2}-\mu^{2}\right) y<0$.

Proof. We evaluate $|f(z+i \lambda)|^{2}-|f(z-i \lambda)|^{2}=\mid\{x+i(y+\lambda)\}^{2}+$ $\left.\Delta^{2}\right|^{2}-\left|\{x+i(y-\lambda)\}^{2}+\Delta^{2}\right|^{2}=8 y \lambda\left(x^{2}+y^{2}+\lambda^{2}-\Delta^{2}\right)$. The assertion directly follows.

Now consider an arbitrary real polynomial $f(z)$ of degree $>0$. It can be written in the form

$$
f(z)=A \prod_{i=1}^{n}\left\{\left(z-a_{i}\right)^{2}+\Delta_{i}^{2}\right\} \prod_{i=1}^{m}\left(z-b_{i}\right)
$$


where $a_{i}, b_{j}$ real; $\Delta_{i}>0, A \neq 0$. Again, let $\lambda$ be a positive number. To any $\Delta_{i}$ exceeding $\lambda$ we construct the circular region $C_{i}$, defined by $\left(x-a_{i}\right)^{2}+$ $y_{i}^{2} \leq \Delta_{i}^{2}-\lambda^{2}$; if $\Delta_{i} \leq \lambda$ we take $C_{i}$ to be empty. By $S=S(f)$ we denote the sum of all $C_{i}$ and the real axis. We now show

Theorem 3. If $f(z)$ is of the type (2.1) and $\lambda>0, \xi$ a complex number $\neq 0$, then all the roots of the polynomial

$$
\xi f(z+i \lambda)+\xi^{*} f(z-i \lambda)
$$

(which has real coefficients) lie in $S$.

Proof. We suppose $\zeta$ to lie in the upper half-plane and outside $S$. Abbreviating (2.1) we write $f(z)=A \prod g_{i}(z) \prod h_{j}(z)$. Trivially $\left|h_{j}(\zeta+i \lambda)\right|>$ $\left|h_{i}(\zeta-i \lambda)\right|$, and it follows from Lemma 1 that also $\left|g_{i}(\zeta+i \lambda)\right|>\left|g_{i}(\zeta-i \lambda)\right|$. Hence $|f(\zeta+i \lambda)|>|f(\zeta-i \lambda)|$. If $\zeta$ lies in the lower half-plane and outside $S$, then $|f(\zeta+i \lambda)|<|f(\zeta-i \lambda)|$. In both cases we conclude that $\zeta$ is not a root of (2.2).

We remark that the limit case of Theorem 3 for $\lambda \rightarrow 0$ leads to a well-known theorem of J. L. W. V. Jensen on the roots of the derivative of a polynomial. (See [3] and [10; Abschn. III, Aufg. 35].)

We want to iterate the result of Theorem 3. Therefore, we first define a set $S_{N}=S_{N}(f), N=1,2, \cdots$, which is the sum of the real axis and the regions $C_{i N}, i=1, \cdots, n$. If $\Delta_{i}>\lambda N^{\frac{1}{2}}$ we take for $C_{i N}$ the region $N^{-1}\left(x-a_{i}\right)^{2}+$ $y^{2} \leq \Delta_{i}^{2}-N \lambda^{2}$, which is bounded by an ellipse; if $\Delta_{i} \leq \lambda N^{\frac{1}{2}}, C_{i N}$ is empty.

It is readily deduced from Theorem 3 that if the roots of the real polynomial $g(z)$ lie in $S_{N}(f)$ then those of $\xi g(z+i \lambda)+\xi^{*} g(z-i \lambda), \xi \neq 0$, lie in $S_{N+1}(f)$.

Theorem 4. Suppose that all the roots of the polynomial $\varphi(u)=\sum_{0}^{N} a_{k} u^{k}$, $a_{N} \neq 0$, lie on the unit circle $|u|=1$, that $f(z)$ is a real polynomial, and that $\lambda>0$. Then the roots of

$$
T^{-N \lambda} \varphi\left(T^{2 \lambda}\right) f(z)=\sum_{k=0}^{N} a_{k} f\{z+(2 k-N) i \lambda\}
$$

are contained in $S_{N}(f)$. Here $T$ represents a translation operator defined by $T^{\mu} f(z)=f(z+i \mu)$.

Proof. The function $u^{-N} \varphi\left(u^{2}\right)$ can be written in the form

$$
u^{-N} \varphi\left(u^{2}\right)=\alpha \prod_{k=1}^{N}\left(\xi_{k} u+\xi_{k}^{*} u^{-1}\right) \quad\left(\alpha \neq 0, \xi_{k} \neq 0\right) .
$$

By Theorem 3, the real polynomial $\left(\xi_{1} T^{\lambda}+\xi_{1}^{*} T^{-\lambda}\right) f(z)$ has its roots in $S_{1}(f)$. A second application shows that the roots of $\left(\xi_{2} T^{\lambda}+\xi_{2}^{*} T^{-\lambda}\right)\left(\xi_{1} T^{\lambda}+\xi_{1}^{*} T^{-\lambda}\right) f(z)$ lie in $S_{2}(f)$, etc., so that the roots of $T^{-N \lambda} \varphi\left(T^{2 \lambda}\right) f(z)$ turn to lie in $S_{N}(f)$.

Theorem 5. Let $f(z)$ be a real polynomial whose roots lie in the strip $|\operatorname{Im} z| \leq$ $\Delta, \Delta \geq 0$, and let $\varphi(u)$ satisfy the conditions stated in Theorem 4 . Then the roots 
of the polynomial (2.3) satisfy $|\operatorname{Im} z| \leq\left\{\Delta^{2}-N \lambda^{2}\right\}^{\frac{1}{3}}$ if $\Delta>\lambda N^{\frac{1}{2}}, \operatorname{Im} z=0$ if $\Delta \leq \lambda N^{\frac{1}{3}}$.

Proof. Follows directly from Theorem 4 and from the definition of $S_{N}(f)$.

3. Application to integral functions. Strong universal factors. Let a real integral function be given of the type

$$
f(z)=A z^{m} e^{a z} \prod_{\nu=1}^{\infty}\left(1-z / \rho_{\nu}\right) e^{z / \rho_{\nu}}
$$

where $A$ is real and $\neq 0, m$ is a natural number, $a$ is real, $\rho_{\nu} \neq 0,\left|\operatorname{Im} \rho_{\nu}\right| \leq \Delta$, $\sum\left|\rho_{\nu}\right|^{-2}<\infty$ and the roots $\rho_{\nu}$ and $\rho_{\nu}^{*}$ have the same multiplicity. It is possible to construct a sequence of polynomials $f_{1}(z), f_{2}(z), \cdots$, all having their roots in the strip $|\operatorname{Im} z| \leq \Delta$, converging uniformly to $f(z)$ in any bounded region. Since the product (3.1) converges uniformly in any bounded region, it is obviously sufficient to prove it for the functions $e^{a z}(a$ real $),\left(1-z / \rho_{\nu}\right) e^{z / \rho_{\nu}}$ if $\rho_{\nu}$ is real and $\left(1-z / \rho_{\nu}\right)\left(1-z / \rho_{\nu}^{*}\right) \exp \left(z / \rho_{\nu}+z / \rho_{\nu}^{*}\right)$ if $\rho_{\nu}$ is not real. In the latter case $\rho_{\nu}^{-1}+\rho_{\nu}^{*-1}$ is real, and thus it only remains to be proved that $e^{a z}$ ( $a$ real) is the uniform limit of a sequence of polynomials with roots only in the $\operatorname{strip}|\operatorname{Im} z| \leq \Delta$. We have indeed $e^{a z}=\lim _{n \rightarrow \infty}(1+a z / n)^{n}$, converging uniformly in any finite region.

Since also $e^{-b z^{2}}(b \geq 0)$ is the limit of a sequence of polynomials with real roots, the same applies to the function $e^{-b z^{2}} f(z), b \geq 0$, if $f(z)$ satisfies the conditions mentioned above.

Conversely, it seems probable that, if a sequence of real polynomials with roots in the strip $|\operatorname{Im} z| \leq \Delta$ converges, uniformly in any bounded region, to an integral function, then this function will be of the type $e^{-b z^{2}} f(z)$, where the genus of $f(z)$ is either 0 or 1 . (The corresponding problem for functions with real roots was solved by Pólya [4].) We do not need the solution of this problem for our present purposes. (After this paper was written the conjecture stated above has been proved by Mr. J. Korevaar.) Namely, we are able to restrict ourselves to integral functions of order $<2$; these functions satisfy $|f(z)|<\exp \left(|z|^{\rho}\right), \rho<2$, for $|z|$ sufficiently large. According to Hadamard's theory such a function can be expanded into a product of the type (3.1) with $\sum\left|\rho_{\nu}\right|^{-2}<\infty$. If we now suppose that the roots of $f(z)$ lie in the strip $|\operatorname{Im} z| \leq$ $\Delta$ and that $f(z)$ is real for real $z$, it immediately follows that $f(z)$ has all the properties mentioned in the beginning of this section. We thus obtain

Theorem 6. If the order of the real integral function $f(z)$ is $<2$ and if the roots of $f(z)$ lie in the strip $|\operatorname{Im} z| \leq \Delta, \Delta \geq 0$, then there exists a sequence of real polynomials $f_{n}(z)$ whose roots lie also in that strip, such that $f_{n}(z) \rightarrow f(z)$ uniformly in any finite region.

For convenience we explicitly formulate the following well-known result (see [10; 123, Abschn. 3, Aufg. 201]). 
TheoRem 7. If $f(z), f_{1}(z), f_{2}(z), \cdots$ are integral functions, $f(z)$ not identically 0 , with $f_{n}(z) \rightarrow f(z)$ uniformly in any finite region, and if the roots of $f_{1}(z), f_{2}(z), \cdots$ all belong to a given closed point-set $S$, then the roots of $f(z)$ also lie in $S$.

Theorems 4 and 5 can now be applied to sequences of polynomials.

Theorem 8. If $f(z)$ satisfies the conditions of Theorem 6 and $\varphi(u)$ those of Theorem 4, then the roots of

$$
T^{-N \lambda} \varphi\left(T^{2 \lambda}\right) f(z) \quad\left(\lambda>0, T^{\mu} f(z)=f(z+i \mu)\right)
$$

satisfy $|\operatorname{Im} z| \leq\left(\Delta^{2}-N \lambda^{2}\right)^{\frac{1}{2}}$ if $\Delta>\lambda N^{\frac{1}{2}}, \operatorname{Im} z=0$ if $0 \leq \Delta \leq \lambda N^{\frac{1}{2}}$.

Proof. Let $f_{n}(z) \rightarrow f(z)$ according to Theorem 6 . It is easily seen that

$$
T^{-N \lambda} \varphi\left(T^{2 \lambda}\right) f_{n}(z) \rightarrow T^{-N \lambda} \varphi\left(T^{2 \lambda}\right) f(z)
$$

uniformly in any finite region. By Theorem 5, the polynomials on the left have their roots in the strip $|\operatorname{Im} z|=\mu=\left\{\operatorname{Max}\left(\Delta^{2}-N \lambda^{2}, 0\right)\right\}^{\frac{1}{2}}$. Now the desired result follows from Theorem 7 .

Theorem 9. Let the real integral function $f(z)$ be of order $<2$ and suppose that $f(z)$ has but a finite number of roots outside the strip $|\operatorname{Im} z| \leq \Delta$. If furthermore $\varphi(u)$ satisfies the conditions of Theorem 4, then all but a finite number of roots of (3.2) satisfy $|\operatorname{Im} z| \leq\left\{\operatorname{Max}\left(\Delta^{2}-N \lambda^{2}, 0\right)\right\}^{\frac{1}{2}}$.

Proof. We put $f(z)=g(z) h(z)$, where $g(z)$ is a polynomial, and the roots of the integral function $h(z)$ lie in the strip $|\operatorname{Im} z| \leq \Delta$. It is easily seen from the arguments used in the beginning of this section that $f$ is the limit of a sequence of polynomials of the type $f_{n}(z)=g(z) h_{n}(z)$, where $h_{n}(z)$ has no roots outside $|\operatorname{Im} z| \leq \Delta$. We may actually take for $h_{n}(z)$ polynomials which have, apart from a number of real ones, only roots which are roots of $h(z)$ also.

According to Theorem 4, the roots of $T^{-N \lambda} \varphi\left(T^{2 \lambda}\right) f_{n}(z)$ lie in $S_{N}\left(f_{n}\right)$. It follows from the definition of $S_{N}$ that $S_{N}\left(f_{n}\right)=S_{N}\left(h_{n}\right)+S_{N}(g) \subseteq R+S_{N}(g)$, where $R$ represents the set $|\operatorname{Im} z| \leq\left\{\operatorname{Max}\left(\Delta^{2}-N \lambda^{2}, 0\right)\right\}^{\frac{1}{2}}$. Now (3.3) and Theorem 7 show that the roots of (3.2) belong to $R+S_{N}(g) . \quad S_{N}(g)$ consists of a finite number of ellipses. Since each ellipse contains but a finite number of roots of (3.2), our proof is completed.

In the special case $N=1, \lambda \geq \Delta$ we can obtain more complete information on the number of non-real roots.

Theorem 9a. If the real integral function $f(z)$ of order $<2$ has exactly $2 k$ roots outside the strip $|\operatorname{Im} z| \leq \Delta$, and if $\lambda \geq \Delta \geq 0, \xi \neq 0$, then the function $f_{1}(z)=\xi f(z+i \lambda)+\xi^{*} f(z-i \lambda)$ has $2 k$ non-real roots at most.

Proof. The function $W(z)=\xi f(z+i \lambda)$ has not more than $k$ roots in the lower half-plane. The theorem now follows by Lemma $2, \S 6$.

In the general case $\lambda<\Delta$ of Theorem 9 the analogue of Theorem $9 \mathrm{a}$ is not true. Even the following statement is false: if $f(z)$ is a real polynomial with 
$2 k$ roots outside the strip $|\operatorname{Im} z| \leq 1$, then $f_{1}(z), 0<\lambda<1$, has at most $2 k$ roots outside the same strip. Taking $\xi=i, \lambda \rightarrow 0$, we should infer that $f^{\prime}(z)$ has at most $2 k$ roots outside that strip. This is incorrect, for instance, for $f(z)=\left(z^{4}+4\right)\left(z^{2}+12^{\frac{1}{2}}\right), f^{\prime}(z)=6 z\left(z^{2}+\frac{1}{3}(12)^{\frac{1}{2}}\right)^{2}$.

In order to be able to apply the preceding results to trigonometric integrals we first state

Theorem 10. Let $b$ be a number $>2$, and let the real or complex function $F(t)$ be integrable over $-\infty<t<\infty$ and satisfy

$$
\begin{gathered}
F(t)=(F(-t))^{*} \text { for all real values of } t, \\
F(t)=O\left(e^{-|t| b}\right)
\end{gathered}
$$$$
(t \rightarrow \pm \infty)
$$

Then the trigonometric integral

$$
f(z)=\int_{-\infty}^{\infty} F(t) e^{i z t} d t
$$

represents a real integral function of order $<2$.

A simple proof can be found in Pólya [8].

Theorem 11. Let $F(t)$ satisfy the conditions of the preceding theorem and suppose that the roots of the function $S(t)=\sum_{-M}^{M} a_{k} e^{k \lambda t}, a_{k}^{*}=a_{-k}, a_{M} \neq 0$, $\lambda>0$, lie on the imaginary axis. Then we have: If the roots (all but a finite number of the roots) of (3.6) lie in the strip $|\operatorname{Im} z| \leq \Delta$ then the roots (all but a finite number of the roots) of the real integral function

$$
\int_{-\infty}^{\infty} F(t) S(t) e^{i z t} d t
$$

lie in the strip $|\operatorname{Im} z| \leq\left\{\Delta^{2}-\frac{1}{2} M \lambda^{2}\right\}^{\frac{1}{2}}$ if $\Delta>\lambda\left(\frac{1}{2} M\right)^{\frac{1}{2}}$, and are real if $\Delta \leq$ $\lambda\left(\frac{1}{2} M\right)^{\frac{1}{2}}$.

Proof. Since the roots of $S(t)$ are purely imaginary, the roots of the polynomial $\varphi(u)=\sum_{-M}^{M} a_{k} u^{M-k}$ lie on the unit circle $|u|=1$; hence $\varphi(u)$ satisfies the conditions of Theorem $4(2 M=N)$. Now our theorem immediately follows from Theorem 8 (Theorem 9) and from the fact that $T^{-\mu} \int_{-\infty}^{\infty} F(t) e^{i z t} d t=$ $\int_{-\infty}^{\infty} F(t) e^{\mu t} e^{i z t} d t$, whence

$$
T^{-N \lambda / 2} \varphi\left(T^{2 \lambda / 2}\right) \int_{-\infty}^{\infty} F(t) e^{i z t} d t=\int_{-\infty}^{\infty} F(t) \sum_{-M}^{M} a_{k} e^{k \lambda t} e^{i z t} d t
$$

A slightly better result can be obtained if $S(t)$ contains factors $\xi e^{\lambda t}+\xi^{*} e^{-\lambda t}$. For instance, the function $S(t)=\xi e^{\lambda t}+2+\xi^{*} e^{-\lambda t}$ gives rise to the strip $|\operatorname{Im} z| \leq\left\{\Delta^{2}-\frac{1}{2} \lambda^{2}\right\}^{\frac{1}{2}}$, but $S(t)=\xi e^{\lambda t}+\xi^{*} e^{-\lambda t}$ gives the strip $|\operatorname{Im} z| \leq$ $\left\{\Delta^{2}-\lambda^{2}\right\}^{\frac{1}{2}}$, which follows from Theorem 8 (Theorem 9) on taking $\varphi(u)=$ $\xi+\xi^{*} u$. By iteration, we obtain 
Theorem 12. If $S(t)=\prod_{1}^{N}\left(\xi_{k} e^{\lambda_{k} t}+\xi_{k}^{*} e^{-\lambda_{k} t}\right)$, where $\left|\xi_{k}\right|=1, \lambda_{k}>0$, $k=1,2, \cdots, N$, and the roots (all but a finite number of the roots) of (3.6) lie in the strip $|\operatorname{Im} z| \leq \Delta$, then the roots (all but a finite number of the roots) of (3.7) lie in the strip $|\operatorname{Im} z| \leq\left\{\operatorname{Max}\left(\Delta^{2}-\sum_{1}^{N} \lambda_{k}^{2}, 0\right)\right\}^{\frac{1}{2}}$.

Theorem 11 proves the statements $(\alpha)$ and $(\beta)$ made in the introduction concerning strong universal factors. Although it will not be used in this paper, we shall prove here that also the functions $e^{\frac{1}{2} \lambda^{2} t^{2}}, \lambda^{2}>0$, have property $(\alpha)$. We do not yet know whether they have or have not property $(\beta)$.

Theorem 13. If $F(t)$ satisfies the conditions of Theorem 10, and if all the roots of (3.6) lie in the strip $|\operatorname{Im} z| \leq \Delta$, then all the roots of $g(z)=\int_{-\infty}^{\infty} F(t) e^{\frac{1}{\lambda^{2} t^{2}} e^{i z t}} d t$ lie in the strip

$$
|\operatorname{Im} z| \leq\left\{\operatorname{Max}\left(\Delta^{2}-\lambda^{2}, 0\right)\right\}^{\frac{1}{2}}
$$

Proof. By Theorem 12, the roots of $g_{N}(z)=\int_{-\infty}^{\infty} F(t)(\cosh \lambda t / N)^{N^{2}} \cdot e^{i z t} d t$ lie in the strip (3.8). Owing to Theorem 7 it is now sufficient to prove that $g_{N}(z) \rightarrow g(z)$ uniformly in any finite region. Now this follows from (3.5) and from the fact that for $\mu^{2}>\lambda^{2}$ we have

$$
e^{-\frac{1}{2} \mu^{2} t^{2}}(\cosh \lambda t / N)^{N^{2}} \rightarrow e^{-\frac{1}{2} \mu^{2} t^{2}+\frac{1}{2} \lambda^{2} t^{2}},
$$

uniformly in $-\infty<t<\infty$. (3.9) results from the inequality cosh $y \leq e^{\frac{1}{2} y^{2}}$, $-\infty<y<\infty$, whence $(\cosh \lambda t / N)^{N^{2}} \leq e^{\frac{1}{2} \lambda^{2} t^{2}}$.

For completeness we mention the following theorem, a slight extension of Pólya's result on universal factors (see [8]) which deals with the case $\Delta=0$.

Theorem 14. Let $F(t)$ satisfy the conditions of Theorem 10 and suppose that the roots of (3.6) lie in the strip $|\operatorname{Im} z| \leq \Delta$. Let $\varphi(z)$ be a real integral function of genus 0 or 1 (that is, a function of the type (3.1)), with real roots only. Then the roots of

$$
\int_{-\infty}^{\infty} F(t) \varphi(i t) e^{i z t} d t
$$

lie in the strip $|\operatorname{Im} z| \leq \Delta$ also.

A proof can be given by introducing quite trivial modifications in Pólya's proof for the case $\Delta=0$. It is, however, also possible to deduce Theorem 14 from Theorem 12 .

It is hardly necessary to say that the function $\varphi(i t) e^{\alpha t^{2}}, \alpha \geq 0$, satisfies as a "universal factor" as well as $\varphi(i t)$, that is to say that the roots of

$$
\int_{-\infty}^{\infty} F(t) \varphi(i t) e^{\alpha t^{2}} e^{i z t} d t
$$

lie in $|\operatorname{Im} z| \leq \Delta$ for any $F(t)$ satisfying the conditions of Theorem 14. But the roots of (3.11) even lie in a narrower strip, which can be shown by applying Theorem 13 to (3.10). 
4. Application of the saddle-point method. An important part in our considerations will be played by the function

$$
H(s)=\int_{0}^{\infty} e^{-g(u)} u^{s} d u
$$

where $\operatorname{Re} s>0$ and

$$
g(u)=u+\alpha_{1} u^{(N-1) / N}+\alpha_{2} u^{(N-2) / N}+\cdots+\alpha_{N} .
$$

We are interested in the asymptotic behavior of $H(s)$ for $\operatorname{Re} s>0,|s|$ large; the natural number $N$ and the coefficients $\alpha_{1}, \cdots, \alpha_{N}$ remaining constant. The $\alpha$ 's need not be real. For $\alpha_{1}=\cdots=\alpha_{N}=0$ we have $H(s)=s \Gamma(s)$, so that asymptotic formulas for the $\Gamma$-function will appear as special cases.

In the sequel, positive constants $a_{1}, a_{2}, \cdots$ will occur, chosen sufficiently large to suit some special purpose. These numbers may depend on $N, \alpha_{1}$, $\cdots, \alpha_{N}$, but not on $s$.

The integrand of (4.1) has, for $s$ large, just one saddle-point $\xi$ in the domain $|\arg u|<\pi$ satisfying

$$
\xi g^{\prime}(\xi)=s .
$$

Namely, putting $s=z^{N}, \xi=w^{N}$ ( $w$ and $z$ are positive for $s$ and $u$ positive), we obtain the equation

$$
w^{N}+(N-1) N^{-1} \alpha_{1} w^{N-1}+(N-2) N^{-1} \alpha_{2} w^{N-2}+\cdots+N^{-1} \alpha_{N-1} w=z^{N},
$$

whence, for $|w|>a_{1}$, the function $1 / z$ can be expanded into a convergent power series $z^{-1}=w^{-1}-(N-1) N^{-2} \alpha_{1} w^{-2}+\cdots$. Solving this equation by the Bürmann-Lagrange inversion formula we obtain $w^{-1}=z^{-1}+\beta_{2} z^{-2}+$ $\beta_{3} z^{-3}+\cdots$ whence $w^{N}=z^{N}\left(1+\beta_{2} z^{-1}+\beta_{3} z^{-2}+\cdots\right)^{-N}=z^{N}\left(1+\gamma_{1} z^{-1}+\right.$ $\left.\gamma_{2} z^{-2}+\cdots\right)$, convergent for $|z|>a_{2}$. It follows that, for $|s|>a_{3}$, the only solutions of (4.3) are

$$
\xi=s\left(1+\gamma_{1} s^{-1 / N}+\gamma_{2} s^{-2 / N}+\cdots\right)
$$

where $s^{-2 / N}, s^{-3 / N}, \cdots$ are derived from one and the same branch of the function $s^{-1 / N}$. We shall restrict ourselves to the regions $|\arg s|<\frac{1}{2} \pi,|\arg u|<\pi$, and hence we only have to consider the case where $s^{1 / N}$ is positive for $s>0$.

We henceforth divide into two cases,

Case $\alpha: 4 \pi / 9<|\arg s|<\frac{1}{2} \pi$,

Case $\beta: \quad|\arg s| \leq 4 \pi / 9$,

and we put $L=\frac{1}{2}|\xi|^{\frac{1}{2}}$ in Case $\alpha, L=\frac{1}{2}|\xi|^{1 / 2 N}$ in Case $\beta, M=|\xi|^{1 / 8 N}, q=$ $\xi-L \xi^{\frac{1}{2}}$ in both cases. (The constant $4 \pi / 9$ is of course not essential. In Case $\beta$ it may be replaced by any other number $<\frac{1}{2} \pi$; in Case $\alpha$ however, it cannot be replaced by arbitrary small positive numbers.) Our integration contour for the integral (4.1) will be

I. A straight line from 0 to $q$.

II. The straight line $u=\xi+y \xi^{\frac{1}{2}},-L \leq y<\infty$; we notice that $\left|\arg \xi^{\frac{1}{2}}\right|<$ $\frac{1}{4} \pi+\delta$ for $|s|$ sufficiently large. 
The major contribution to $H(s)$ is furnished by the integral along II passing through the saddle-point $\xi$. Its value is

$$
\int_{a}^{\infty} e^{-o(u)} u^{s} d u=\xi^{\frac{1}{2}} \int_{-L}^{\infty} e^{-\kappa(u)} d y
$$

where $K(u)=g(u)-s \log u$ and $u=\xi+y \xi^{\frac{1}{2}}$. We have

$$
\begin{aligned}
d K / d y & =\left\{g^{\prime}(u)-s u^{-1}\right\} \xi^{\frac{1}{2}}=u^{-1} \xi^{\frac{1}{2}}\left\{u g^{\prime}(u)-\xi g^{\prime}(\xi)\right\} \\
& =u^{-1} \xi^{\frac{1}{2}} \int_{0}^{y}\left(d\left\{u g^{\prime}(u)\right\} / d y\right) d y=u^{-1} \xi \int_{0}^{y}\left(d\left\{u g^{\prime}(u)\right\} / d u\right) d y .
\end{aligned}
$$

It is easily seen from (4.4) that, for $|s|>a_{4}, \operatorname{Re} s>0, y \geq-L$, we have

$$
|u|>\frac{1}{3}|\xi|, \quad\left|\left(d\left\{u g^{\prime}(u)\right\} / d u\right)-1\right|<a_{5}|\xi|^{-1 / N},
$$

$$
|\arg (u / \xi)|<3 \pi / 8 .
$$

It follows from (4.6), (4.7), (4.8) that, if $|s|>a_{6}$, Re $K(u)$ decreases from $y=-L$ to $y=-M$. Furthermore, for $y>M$ we have, again by (4.6), (4.7) and (4.8), for $|s|>a_{7}$,

$$
\operatorname{Re}(d K / d y)>\frac{1}{4}|\xi / u| y>\frac{1}{4} y /(1+y)>\frac{1}{8} .
$$

It follows that

$$
\begin{gathered}
\left|\int_{-L}^{-M} e^{-K(u)} d y\right|<\frac{1}{2}|\xi|^{\frac{1}{3}}\left|\exp \left(-K\left(\xi-M \xi^{\frac{1}{3}}\right)\right)\right|, \\
\left|\int_{M}^{\infty} e^{-K(u)} d y\right|<8\left|\exp \left(-K\left(\xi+M \xi^{\frac{1}{3}}\right)\right)\right| .
\end{gathered}
$$

We now consider the interval $-M \leq y \leq M$. Since $M=\xi^{1 / 8 N}$, we have $\left|\xi u^{-1}-1+y \xi^{-\frac{1}{2}}\right|<a_{8} y^{2}|\xi|^{-1},|s|>a_{9}$, and by (4.6) and (4.7) we have

$$
\left|d K / d y-y+y^{3} \xi^{-\frac{1}{2}}\right|<a_{10}\left(\left|y \xi^{-1 / N . N}\right|+\left|y^{3} \xi^{-1}\right|\right) \quad\left(|s|>a_{11}\right) .
$$

Hence, for $-M \leq y \leq M$,

$$
\left|K(u)-K(\xi)-\frac{1}{2} y^{2}+\frac{1}{3} y^{3} \xi^{-\frac{1}{2}}\right|<a_{10}\left(\left|y^{2} \xi^{-1 / N}\right|+\left|y^{4} \xi^{-1}\right|\right),
$$

$\int_{-M}^{M} e^{-K(u)} d y$

$$
\begin{aligned}
& =e^{-K(\xi)} \int_{-M}^{M} e^{-\frac{1}{2} \nu^{2}}\left\{1+\frac{1}{3} y^{3} \xi^{-\frac{1}{2}}+O\left(\frac{y^{6}}{|\xi|}+\frac{y^{2}}{|\xi|^{1 / N}}+\frac{y^{4}}{|\xi|}\right)\right\} d y \\
& =e^{-K(\xi)}\left\{(2 \pi)^{\frac{1}{2}}+O\left(e^{-\frac{1}{2} M^{2}}\right)+0+O\left(\frac{1}{|\xi|}+\frac{1}{|\xi|^{1 / N}}+\frac{1}{|\xi|}\right)\right\} \\
& =(2 \pi)^{\frac{1}{2}} e^{-K(\xi)}\left\{1+O\left(\xi^{-1 / N}\right)\right\} .
\end{aligned}
$$


From (4.12) we easily infer that the right members of (4.10) and (4.11) are $O\left(\xi^{-1 / N} e^{-K(\xi)}\right)$, so that (4.5), (4.10), (4.11) and (4.13) give

$$
\int_{a}^{\infty} e^{-o(u)} u^{s} d u=(2 \pi \xi)^{\frac{1}{2}} e^{-K(\xi)}\left\{1+O\left(\xi^{-1 / N}\right)\right\} .
$$

In the second place we consider the integral

$$
\int_{0}^{a} e^{-o(u)} u^{s} d u
$$

for which the Cases $\alpha$ and $\beta$ have to be treated separately.

Case $\alpha$. Since $q=\xi-\frac{1}{2}|\xi|^{\frac{1}{1}} \xi^{\frac{1}{2}}$ and $4 \pi / 9<\arg s<\frac{1}{2} \pi$, it is easily seen, on drawing a figure and carrying out some elementary trigonometric calculations, that $|\arg q|>\left|\arg \left(e^{4 \pi i / 9}-\frac{1}{2} e^{2 \pi i / 9}\right)\right|-\epsilon>5 \pi / 9$ for $|s|$ large. It follows that, if $|s|>a_{12}$ and if $u$ runs through the straight line from 0 to $q$, the maximum value of $\left|e^{-o(u)}\right|$ is attained at $u=q$. The same being true for $\left|u^{s}\right|$ (since $\operatorname{Re} s>0$ ), we obtain

$$
\left|\int_{0}^{a} e^{-o(u)} u^{s} d u\right|<\left|q e^{-K(a)}\right| .
$$

It was noticed before that, if $|s|>a_{6}, \operatorname{Re} K(u)$ decreases from $u=q$ to $u=$ $\xi-M \xi^{\frac{1}{2}}$, so that $\operatorname{Re} K(q) \geq \operatorname{Re} K\left(\xi-M \xi^{\frac{1}{2}}\right)$, whence the left side of (4.16) is $O\left(\xi^{-1 / N} e^{-K(\xi)}\right)$ (see (4.12)).

Case $\beta$. $|\arg s| \leq 4 \pi / 9$. We put $u=q t, 0 \leq t \leq 1, q=\xi-\frac{1}{2}|\xi|^{1 / 2 N} \xi^{\frac{1}{2}}$. Then we have (see (4.6))

$$
d K / d t=q\left\{g^{\prime}(u)-s / u\right\}=t^{-1}\left\{u g^{\prime}(u)-\xi g^{\prime}(\xi)\right\},
$$

and $u g^{\prime}(u)-\xi g^{\prime}(\xi)=\int_{\xi}^{u}\left\{u g^{\prime}(u)\right\}^{\prime} d u,\left\{u g^{\prime}(u)\right\}^{\prime}=1+O\left(u^{-1 / N}\right)$. Hence $a_{13}$ and $a_{14}$ can be chosen such that for $|u|>a_{13},|s|>a_{14}$,

$$
u g^{\prime}(u)-\xi g^{\prime}(\xi)=-(1+\eta)(\xi-u) \quad(|\eta|<\sin \pi / 20) .
$$

Now it is easily seen from a figure that for $|s|>a_{15},|\arg (\xi-u)|<$ $|\arg \xi|<9 \pi / 20$. So it follows from (4.17) and (4.18) that, for $|s|>a_{16}, \operatorname{Re}$ $K(u)$ decreases if $u$ runs through the straight line segment from $u=u_{0}=$ $a_{13} q /|q|$ to $u=q$, whence $\left|e^{-K(u)}\right| \leq\left|e^{-K(\alpha)}\right|$ for $u$ on that segment.

Let $\mu$ be the maximum value of $\left|e^{-o(u)}\right|$ on the remaining segment from 0 to $u_{0}$. If $u$ lies between 0 and $u_{0}, 0 \leq t \leq a_{13}|q|^{-1}$, we have

$$
\left|e^{-K(u)}\right| \leq \mu\left|e^{o\left(u_{0}\right)}\left(u / u_{0}\right)^{s} e^{-K\left(u_{0}\right)}\right| \leq \mu\left|e^{o\left(u_{0}\right)}\right|\left|e^{-K(\alpha)}\right| .
$$

Since the constant $a_{16}=\mu\left|e^{o\left(u_{0}\right)}\right|$ is independent of $s$, we obtain

$$
\left|\int_{0}^{a} e^{-o(u)} u^{s} d u\right|<\left(1+a_{16}\right)\left|q e^{-K(a)}\right| .
$$

Just as in Case $\alpha$, we infer that (4.15) is $O\left(\xi^{-1 / N} e^{-K(\xi)}\right)$. 
TheORem 15. If $b$ is a positive constant and $H(s)$ is given by (4.1), we have

$$
H(s)=(2 \pi \xi)^{\frac{1}{2}} e^{-o(\xi)} \xi^{s}\left\{1+O\left(s^{-1 / N}\right)\right\},
$$

uniformly for $\operatorname{Re} s>-b,|s| \rightarrow \infty$. Here $\xi=s+\gamma_{1} s^{1-1 / N}+\gamma_{2} s^{1-2 / N}+\cdots$ (absolutely convergent for s large) satisfies $\xi g^{\prime}(\xi)=s$.

(We notice that for $N=1$ we obtain $g^{\prime}(u)=1, \xi=s$ and then (4.20) becomes the familiar Stirling formula for $\Gamma(s+1)$.)

Proof. For Re $s>0$ the result follows from (4.14), (4.16), and (4.19). In order to be able to consider values of $s$ in the left half-plane, we continue $H(s)$ by the formula

$$
\begin{gathered}
(s+1) H(s)=H(s+1)+\alpha_{1}(N-1) N^{-1} H\left(s+1-N^{-1}\right) \\
+\cdots+\alpha_{N-1} N^{-1} H\left(s+N^{-1}\right),
\end{gathered}
$$

which can be found by partial integration: $(s+1) H(s)=\int_{0}^{\infty} e^{-o(u)} g^{\prime}(u) \cdot u^{s+1} d u$. It follows from (4.21) that $H(s)$ can be continued over the whole plane but for the points $s=-1,-1-1 / N,-1-2 / N, \cdots$, which are possible poles of $H(s)$. It also follows that (4.21) holds for all values of $s$ except for these points.

The function $h(s)=(2 \pi \xi)^{\frac{1}{2}} e^{-o(\xi)} \xi^{s}, \xi=s+\gamma_{1} s^{1-1 / N}+\cdots$, is regular and satisfies

$$
s^{\rho} h(s-\rho) / h(s)=1+O\left(s^{-1 / N}\right)
$$

uniformly for $0 \leq \rho \leq b+1,|\arg s|<\pi,|s|>a_{17}$. This can be shown by some elementary calculations. Now suppose that (4.20) is true for the region $\operatorname{Re} s>-k / N$. Then, by (4.21) and (4.22), it can be verified for $\operatorname{Re} s>$ $-(k+1) / N$. Hence the theorem follows by induction.

In the following Theorems 16 and 17 we shall collect some results concerning $H(s)$ to be used in the next section.

Theorem 16. Put $s=\sigma+i \tau$ ( $\sigma$ and $\tau$ real), and let $b$ and $c$ be positive constants. There are constants $A$ and $C$ ( $A$ and $C$ may depend on $\left.\alpha_{1}, \cdots, \alpha_{N}\right)$ such that, for $-b<\sigma<c|\tau|,|\tau|>A$ and any positive number $\rho$ we have

$$
|H(s-\rho)|<C^{1+\rho}|s|^{-\rho}|H(s)| \text {. }
$$

Proof. Take $C=\left\{1+\left|\alpha_{1}\right|(1-1 / N)+\cdots+\left|\alpha_{N-1}\right| / N\right\}\left(1+c^{2}\right)^{\frac{1}{2}}$. It follows from Theorem 15 and from (4.22), that $A, A>C$, can be determined such that for $0 \leq \rho \leq 1, \sigma \geq-b,|\tau|>A$, we have $\left|s^{\rho} H(s-\rho) / H(s)\right|<$ $\left(1+c^{2}\right)^{\frac{1}{2}}<C^{1+\rho}$. Now the case $\rho>1$ can be proved by induction. Suppose that (4.23) is true for $0 \leq \rho \leq k / N$ ( $k$ an integer, $k \geq N)$, and that $k / N<$ $\rho_{1} \leq(k+1) / N$. Then we have $\left|H\left(s-\rho_{1}+1\right)\right|<C^{\rho_{1}}|H(s)| /|s|^{\rho_{1}-1}$, $\left|H\left(s-\rho_{1}+1-1 / N\right)\right|<C^{\rho_{1}+1 / N}|H(s)| /|s|^{\rho_{1}-1+1 / N}$, and so on, and hence, since $|\tau|>A>C,\left|H\left(s-\rho_{1}+1-k / N\right)\right|<C^{\rho_{1}}|s|^{1-\rho_{1}}|H(s)|, k=1,2$, $\cdots, N-1$. It now follows from (4.21) that 
$\left|\left(s-\rho_{1}+1\right) H\left(s-\rho_{1}\right)\right|$

$$
\leq\left(1+\left|\alpha_{1}\right|(N-1) N^{-1}+\cdots+\left|\alpha_{N-1}\right| N^{-1}\right) C^{\rho_{1}}|s|^{1-\rho_{1}}|H(s)| .
$$

Since $\sigma<c|\tau|$ and $\rho_{1}>1$, we have $\left|s-\rho_{1}+1\right| \geq|s|\left(1+c^{2}\right)^{-\frac{1}{2}}$ and (4.23) follows for $\rho=\rho_{1}$.

Theorem 17. If $b, c$ and $\delta$ are given positive numbers, then positive numbers $A$ and $C$ can be found such that $|H(s)|>C e^{-\left(\frac{1}{2} \pi+\delta\right)|\tau|+c \sigma}, s=\sigma+i \tau$, in the region $\sigma>-b,|s|>A$.

This follows from Theorem 15 by some simple calculations.

5. Proof of Theorems 1 and 2. In order to complete the proof for Theorem 2 , which was outlined in the introduction, it is sufficient to show (Theorem 18) that the integral (1.8) has but a finite number of roots outside any strip $|\operatorname{Im} z|<$ $\epsilon, \epsilon>0$. Theorem 2 follows (for $Q(t)$ ) by applying Theorem 18 to the function $Q_{1}(t)=Q(t) /\left(e^{t}+2+e^{-t}\right)$ which also satisfies the conditions of Theorem 2, and using Theorem 12 or 11.

We shall establish a series expansion (formula (5.7)) for $\Phi(z)$ which generalizes a formula of Polya (see [6; formula (11)]) for the function (1.2). The occurrence of $Q(t)$ in our $\Phi(z)$ makes it very difficult to carry out Pólya's method in the present case. We therefore develop a new method which uses contour integration.

Let the numbers $\alpha_{1}, \alpha_{2}, \cdots, \alpha_{N}$ be defined by $p_{N} y^{N}+p_{N-1} y^{N-1}+\cdots+$ $\frac{1}{2} p_{0} \equiv\left(p_{N}^{1 / N} y\right)^{N}+\alpha_{1}\left(p_{N}^{1 / N} y\right)^{N-1}+\cdots+\alpha_{N}$ and define $g(u)$ by (4.2). Then we have, by (1.6),

$$
P(t)=g\left(p_{N} e^{N t}\right)+g^{*}\left(p_{N}^{*} e^{-N t}\right) .
$$

Let $B>0$ be such that $Q(t)$ is regular for $|\operatorname{Re} t| \geq B$. Consider the following paths $W_{1}, W_{2}, W_{3}$ in the complex $t$-plane.

$W_{1}$ consists of a half line from $2 \pi i+\infty$ up to the point $2 \pi i+B$, the line segment from $2 \pi i+B$ to $B$, and the real axis from $B$ to $+\infty$.

$W_{2}$ is the contour of a rectangle, taken in positive direction, with vertices $B$, $2 \pi i+B, 2 \pi i-B,-B$.

$W_{3}$ consists of the segments $-\infty$ to $-B,-B$ to $2 \pi i-B$ and $2 \pi i-B$ to $2 \pi i-\infty$.

Considering $e^{-P(t)} Q(t) e^{i z t}$ as our integrand, we immediately find that

$$
\int_{W_{1}}+\int_{W_{2}}+\int_{W_{3}}=\int_{-\infty}^{\infty}-\int_{2 \pi i-\infty}^{2 \pi i+\infty}=\left(1-e^{-2 \pi z}\right) \Phi(z) .
$$

For $t$ on $W_{1}$, and $B$ sufficiently large, we have an expansion

$$
\left(\exp \left(-g^{*}\left(p_{N}^{*} e^{-N t}\right)\right)\right) Q(t)=\sum_{\nu=-K}^{\infty} \beta_{\nu} e^{-\nu t} \quad\left(\beta_{-K} \neq 0\right)
$$


converging absolutely and uniformly with respect to $t$. Hence

$$
\int_{W_{1}}=\sum_{-K}^{\infty} \beta_{\nu} \int_{W_{1}}\left(\exp \left(-g\left(p_{N} e^{N t}\right)\right)\right) e^{-v t+i z t} d t .
$$

The integral

$$
\int_{W_{1}}\left(\exp \left(-g\left(p_{N} e^{N t}\right)\right)\right) e^{i z t} d t
$$

converges for any complex value of $z$. To evaluate it, first suppose $\operatorname{Im} z<0$, then shift the vertical part of the path $W_{1}$ infinitely to the left. It follows that (5.5) equals

$$
\left(1-e^{-2 \pi z}\right) \int_{-\infty}^{\infty}\left(\exp \left(-g\left(p_{N} e^{N t}\right)\right)\right) e^{i z t} d t
$$

and hence

$$
\int_{W_{1}}\left(\exp \left(-g\left(p_{N} e^{N t}\right)\right)\right) e^{i z t} d t=N^{-1}\left(1-e^{-2 \pi z}\right) p_{N}^{-i z / N} H\left(i z N^{-1}-1\right),
$$

where $H$ is the function introduced in (4.1). Since $H(s)$ is regular over the whole plane, with exception of simple poles at $s=-k / N, k=1,2, \cdots$, the right side of (5.6) is an integral function, and (5.6) holds for all $z$.

We can deal with $\int_{w_{z}}$ in the same way, and after that (5.2) and (5.3) lead to expansion

$$
\begin{aligned}
\Phi(z)=\frac{1}{N} \sum_{\nu=-K}^{\infty} \beta_{\nu} p_{N}^{(-i z+\nu) / N} H\left(\frac{i z-\nu}{N}-1\right) \\
+\frac{1}{N} \sum_{\nu=-K}^{\infty} \beta_{\nu}^{*} p_{N}^{*(i z+\nu) / N} H^{*}\left(\frac{-i z-\nu}{N}-1\right) \\
\quad+\frac{1}{1-e^{-2 \pi z}} \int_{W} e^{-P(t)} Q(t) e^{i z t} d t=\Phi_{1}(z)+\Phi_{3}(z)+\Phi_{2}(z)
\end{aligned}
$$

This formula holds for all values of $z$, with the exception of the points $z=i k$, $k=0, \pm 1, \pm 2, \cdots$.

We are now able to prove

Theorem 18. If $P(t)$ and $Q(t)$ satisfy the conditions of Theorem 2, and if we put

$$
\varphi(z)=N^{-1} \beta_{-K} p_{N}^{-(i z+K) / N} H\left((i z+K) N^{-1}-1\right),
$$

then we have, for $\epsilon>0$,

$$
\Phi(z)=\varphi(z)\{1+o(1)\}
$$

uniformly in the half-plane $\operatorname{Im} z \leq-\epsilon$, and $\Phi(z)$ has but a finite number of roots in that half-plane. Since $(\Phi(z))^{*}=\Phi\left(z^{*}\right)$, an analogous result holds for $\operatorname{Im} z \geq \epsilon$. 
Proof. We first consider the point-set $R$ defined by the inequalities

$$
-|\operatorname{Re} z|<\operatorname{Im} z<-\epsilon \quad(|z|>A) .
$$

(On generalizing Theorem 16 it is possible to extend our considerations to the regions defined by the set of inequalities $|\operatorname{Re} z|>1$, Im $z<-\epsilon,|z|>A$. The power of the Phragmén-Lindelöf theorem applied below however enables us to restrict ourselves to the smaller regions indicated above.) Here $A$ is chosen sufficiently large to suit some conditions indicated in the following.

By Theorem 16, the first series of (5.7) satisfies

$$
\begin{aligned}
\left|\Phi_{1}(z)-\varphi(z)\right| & \leq \sum_{-K+1}^{\infty}\left|\beta_{\nu} \beta_{-K}^{-1} p_{N}^{(K+\nu) / N} C_{1}^{1+\nu / N} z^{-(K+\nu) / N} \varphi(z)\right| \\
& <C_{2}|z|^{-1 / N}|\varphi(z)|
\end{aligned}
$$

if $A$ is sufficiently large and $z \varepsilon R . \quad\left(C_{1}, C_{2}, \cdots\right.$ may depend on $N, K, p_{i}$, $\beta_{i}$, but not on $z$.) Analogously

$$
\left|\Phi_{3}(z)-\varphi^{*}(-z)\right|<C_{3}|z|^{-1 / N}\left|\varphi^{*}(-z)\right| \text {. }
$$

Furthermore we have, again by Theorem 16, taking $\rho=\left(i z-i z^{*}\right) / N$,

$$
\left|H^{*}\left(\frac{-i z+K}{N}-1\right)\right|=\left|H\left(\frac{i z^{*}+K}{N}-1\right)\right|<C_{4}|z|^{-2 \epsilon / N}\left|H\left(\frac{i z+K}{N}-1\right)\right|
$$

for $z \varepsilon R$ and $A$ sufficiently large. Hence, by (5.11) and (5.12)

$$
\Phi_{1}(z)+\Phi_{3}(z)=\varphi(z)\{1+o(1)\} .
$$

We now turn to the third part of (5.7), i.e., the function $\Phi_{2}(z)$. It follows from the regularity properties of $Q(t)$ (see Theorem 2) that the path $W_{2}$ can be reduced to the path $W_{2}^{\sim}$, consisting of a rectangle with vertices $B+\left(\frac{1}{2} \pi+\delta_{1}-\right.$ $\left.\arg p_{N}\right) i / N ; B+2 \pi i+\left(-\frac{1}{2} \pi-\delta_{1}-\arg p_{N}\right) i / N ;-B+2 \pi i+\left(-\frac{1}{2} \pi-\delta_{1}-\right.$ $\left.\arg p_{N}\right) i / N ;-B+\left(\frac{1}{2} \pi+\delta_{1}-\arg p_{N}\right) i / N$ for a certain positive number $\delta_{1}$. The rectangle being independent of $z$, we find, for $\operatorname{Im} z<-\epsilon$,

$$
\begin{aligned}
\left|\Phi_{2}(z)\right| & =\left|\left(1-e^{-2 \pi z}\right)^{-1} \int_{W_{2}^{\infty}}\right| \\
& \leq C_{5} \exp \left\{B|\operatorname{Im} z|-\left(\frac{1}{2} \pi+\delta_{1}\right)|\operatorname{Re} z / N|+\arg p_{N} \operatorname{Re} z / N\right\} .
\end{aligned}
$$

From Theorem 17, with $c>B N, \delta<\delta_{1}$, we now easily derive that (see (5.8))

$$
\Phi_{2}(z)=\varphi(z) o(1)
$$

uniformly in the region considered.

It follows from (5.13) and (5.14) that (5.9) holds uniformly for $z \varepsilon R$.

In the region $R_{1}$ defined by $|\operatorname{Im} z|<-|\operatorname{Re} z|,|z|>A$, we have, by Theorem 10, $\Phi(z)=O\left(\exp |z|^{\lambda}\right), \lambda<2$. On the boundary of $R_{1}$ we have 
$\Phi(z)=\varphi(z)\{1+o(z)\}$ and furthermore $|\varphi(z)|>1$ for $z$ in $R_{1}$, provided that $A$ has been chosen sufficiently large. It follows, by a well-known theorem of Phragmén-Lindelöf, that (5.9) holds uniformly in $R_{1}$.

Since $H(s)$ has only a finite number of roots for $\operatorname{Re} s>-b$, which follows from Theorem 17, now Theorem 18 is completely proved. Hence Theorem 2 is true (see Introduction).

We now turn to the proof of Theorem 1 . In the first place we deduce from Theorem 2 and Theorem 11, by an argument explained in the introduction,

Theorem 19. If $P(t)=\sum_{-N}^{N} p_{k} e^{k t}\left(N>0\right.$, Re $\left.p_{N}>0, p_{k}^{*}=p_{-k}\right)$ and if all the.roots of its derivative $P^{\prime}(t)$ are purely imaginary, then the function $\int_{-\infty}^{\infty} e^{-P(t)} e^{i z t}$ dt has real roots only.

We easily infer

THEOREM 20. If all the roots of the derivative of the polynomial $f(t)=\sum_{1}^{N}$ $q_{n} t^{n}$ ( $N$ positive and even) are purely imaginary, and if $q_{N}>0$ (so that $f(t)$ is real for $t$ purely imaginary), then the function $\Psi(z)=\int_{-\infty}^{\infty} e^{-f(t)} e^{i z t} d t$ has real roots only.

Proof. We put $f^{\prime}(t)=N q_{N} \prod_{\nu=1}^{N-1}\left(t-i \rho_{\nu}\right), \rho_{\nu}$ real, and for $\lambda>0$,

$$
N q_{N} \prod_{\nu=1}^{N-1} \lambda \sinh \left(t-i \rho_{\nu}\right) \lambda^{-1}=\varphi_{\lambda}(t), \quad \int_{0}^{t} \varphi_{\lambda}(\tau) d \tau=\psi_{\lambda}(\mathrm{t}) .
$$

$\varphi_{\lambda}(t)$ has the form

$$
\sum_{-N+1}^{N-1} c_{k} e^{k t / \lambda}
$$

Since $N$ is even we have $c_{0}=0$; hence $\psi_{\lambda}(t)$ also has the form (5.15). It is easily verified that $\psi_{\lambda}(t)$ is real for purely imaginary values of $t$, and furthermore

$$
\psi_{\lambda}(\lambda t)=2 N(N-1)^{-1}\left(\frac{1}{2} \lambda\right)^{N} q_{N} e^{-i \Sigma \rho_{\nu} / \lambda} e^{(N-1) t}+\cdots .
$$

It follows, that, for $\lambda>\lambda_{0}=2 \pi^{-1}\left|\sum_{1}^{N-1} \rho_{\nu}\right|$, the function $P_{\lambda}(t)=\psi_{\lambda}(\lambda t)$ satisfies the conditions of Theorem 19 , so that $\Phi_{\lambda}(z)=\int_{-\infty}^{\infty} e^{-\psi_{\lambda(t)}} e^{i z t} d t$ has real roots only. It remains to be proved that (see Theorem 7)

$$
\lim _{\lambda \rightarrow+\infty} \Phi_{\lambda}(z)=\Psi(z)
$$

uniformly in any bounded domain of the $z$-plane.

We can determine positive constants $\delta_{1}<\pi / 2 N$ and $\delta_{2}$ such that

$$
|(\sinh w) / w|>\frac{1}{2}, \quad|\arg (\sinh w) / w|<1 / 2 N,
$$

for any value of $w$ satisfying $|\arg w|<\delta_{1}$ and $|\operatorname{Im} w|<\delta_{2}$. Furthermore we can fix positive numbers $A_{1}$ and $A_{2}$ such that the numbers $w=\left(t-i \rho_{\nu}\right) / \lambda$ lie in that region for all values of $t>A_{1}, \lambda>A_{2}$ and $\nu, 1 \leq \nu \leq N-1$. It 
now follows from (5.17) that a positive constant $c$ exists such that $\operatorname{Re} \varphi_{\lambda}(t)>$ $c t^{N-1}$ for $t>A_{1}, \lambda>A_{2}$. Hence

$$
\lim _{A \rightarrow \infty} \limsup _{\lambda \rightarrow \infty}\left|\int_{A}^{\infty} e^{-\psi_{\lambda}(t)} e^{i z t} d t\right|=0
$$

uniformly in any bounded domain of the $z$-plane. The same holds, of course, for $\int_{-\infty}^{-A}$.

Since $q_{N}>0, N$ even, we also find that

$$
\lim _{A \rightarrow \infty}\left|\int_{A}^{\infty} e^{-f(t)} e^{i z t} d t\right|=0
$$

uniformly in any bounded domain, and the same for $\int_{-\infty}^{-A}$. For any fixed value of $A$ we have

$$
\lim _{\lambda \rightarrow \infty} \int_{-A}^{A} e^{-\psi_{\lambda}(t)} e^{i z t} d t=\int_{-A}^{A} e^{-f(t)} e^{i z t} d t
$$

uniformly in any bounded domain, because $\psi_{\lambda}(t) \rightarrow f(t)$ uniformly in $-A \leq$ $t \leq A$.

From (5.18), (5.19) and (5.20) we infer (5.16) and our theorem is proved.

Proof of Theorem 1. Suppose that $f(t)$ satisfies the conditions of Theorem 1. These conditions can also be expressed in the following form (see [4]): $f^{\prime}(t)$ is of the type $f^{\prime}(t)=a e^{b t^{2}} t^{2 k+1} \prod_{\nu=1}^{\infty}\left(1+\delta_{\nu}^{2} t^{2}\right)$ where $a>0, b \geq 0, k$ an integer $\geq 0, \delta_{\nu} \geq 0, \nu=1,2, \cdots, \sum_{1}^{\infty} \delta_{v}^{2}<\infty$. Now let $f_{n}(t)$ be defined by $f_{n}^{\prime}(t)=$ $a\left(1+b t^{2} / n\right)^{n} t^{2 k+1} \prod_{1}^{n}\left(1+\delta_{\nu} t^{2}\right), f_{n}(0)=f(0)$ and put $\Phi(z)=\int_{-\infty}^{\infty} e^{-f(t)} e^{i z t} d t$, $\Phi_{n}(z)=\int_{-\infty}^{\infty} e^{-f_{n}(t)} e^{i z t} d t$. It is easy to find positive numbers $A, \epsilon$ and $n_{0}$, such that for $n>n_{0}, t>A$ or $t<-A$ we have $f(t)>\epsilon t^{2}$ and $f_{n}(t)>\epsilon t^{2}$.

Since $f_{n}(t) \rightarrow f(t)$ for $n \rightarrow \infty$, uniformly in any finite $t$-interval, we now easily infer that (see proof of Theorem 20)

$$
\lim _{n \rightarrow \infty} \Phi_{n}(z)=\Phi(z)
$$

uniformly in any bounded domain of the $z$-plane.

It is easily seen that the polynomials $f_{n}(z)$ satisfy the conditions of Theorem 20 , whence it follows that $\Phi_{n}(z)$ has real roots only. Application of (5.21) and Theorem 7 completes the proof of Theorem 1 .

6. Functions of the type (1.9). We consider the functions of the type (1.9). In connection with the Riemann hypothesis (see \$7) it may be important to investigate classes of functions of this type.

In the following we shall prove some results concerning these functions. Very little of the preceding sections will be needed here, since the reality of the roots of $\int_{-\infty}^{\infty} C(t) e^{i z t} d t, C(t)=\exp (-\lambda \cosh t)$, (special case of Theorem 1 or 19) was already proved by Pólya (see (1.2)). Only Theorem 25 requires asymptotic expressions and strong universal factors as its tools.

First we prove 
Lemma 2. If $U(z)$ and $V(z)$ are real polynomials such that $W(z)=U(z)+$ $i V(z)$ has $n$ roots in the lower half-plane, then $U(z)$ has $n$ pairs of conjugate complex roots at most.

(The case $n=0$ is the well-known theorem of Hermite-Biehler (see [10; Abschn. 3, Aufg. 25]).)

Proof. We may assume that $U(z)$ and $V(z)$ have no real roots in common, so that $W(z)$ has no real roots. We also assume that the degree $m$ of $W(z)$ satisfies $m \geq 2 n$, for otherwise the result is trivial. Now if $z$ runs through the real axis from $-\infty$ to $\infty$, the argument of $W(z)$ increases by an amount of $\pi(m-2 n)$. Hence there are at least $m-2 n$ different points on the real axis where $W(z)$ is purely imaginary. If $z=\infty$ is not one of these points, we thus find $m-2 n$ real roots of $U(z)$ at least, and otherwise at least $m-2 n-1$. But in the last case the degree of $U(z)$ must be $<m$, so that $U(z)$ has at most $2 n$ complex roots in both cases.

TheoRem 21. The function (1.9) (which has but a finite number of non-real roots on account of Theorem 2) has $N$ pairs of conjugate complex roots at most.

Proof. The functions, where $C(t)=\exp (-\lambda \cosh t), \lambda>0$,

$$
\begin{gathered}
\Phi(z)=\int_{-\infty}^{\infty} C(t) e^{i z t} d t \\
\Phi_{1}(z)=\int_{-\infty}^{\infty}(C(t) \cosh t) e^{i z t} d t, \Phi_{2}(z)=\int_{-\infty}^{\infty}(C(t) i \sinh t) e^{i z t} d t
\end{gathered}
$$

have real roots only (Theorems 1 and 11), whereas the roots of $\Phi(z-i)=$ $\Phi_{1}(z)-i \Phi_{2}(z)$ lie in the upper half-plane.

By partial integration it is easily seen that, for $k=0,1,2, \cdots$, the functions $z^{k} \Phi_{1}(z)$ and $z^{k} \Phi_{2}(z)$ are of the form

$$
z^{k} \Phi_{l}(z)=\int_{-\infty}^{\infty} C(t) \sum_{n=-k-1}^{k+1} a_{n, k}^{(l)} e^{n t} e^{i z t} d t \quad(l=1,2)
$$

where $a_{n, k}^{*(l)}=a_{-n, k}^{(l)}$ and the highest coefficients $a_{k+1, k}^{(l)}, l=1,2$, are $\neq 0$. Furthermore $a_{n, k}^{(l)}$ is real if $k+l$ is odd, and purely imaginary if $k+l$ is even. It follows that the function $\Psi(z)$, given by (1.9), can be expressed as a linear combination of $\Phi(z), \Phi_{1}(z), z \Phi_{1}(z), \cdots, z^{N-1} \Phi_{1}(z), \Phi_{2}(z), \cdots, z^{N-1} \Phi_{2}(z)$, with real coefficients. Since $z \Phi(z)=-\lambda \Phi_{2}(z)$ we have

$$
z \Psi(z)=A(z) \Phi_{1}(z)+B(z) \Phi_{2}(z),
$$

where $A(z)$ and $B(z)$ are real polynomials of degree $N$ at most.

Now if $F(z)$ and $G(z)$ are real polynomials, of arbitrary degree, with the property that the roots of $F(z)-i G(z)$ all lie in the upper half-plane, then $A(z) F(z)+B(z) G(z)$ has at most $N$ pairs of conjugate complex roots. This follows by application of Lemma 2 to the function $U(z)+i V(z)=(A(z)+$ 
$i B(z))(F(z)-i G(z))$. On approximating $\Phi(z-i)=\Phi_{1}(z)-i \Phi_{2}(z)$ by polynomials $F(z-i)$ whose roots also lie in the upper half-plane, we find that (6.3) has $N$ pairs of conjugate complex roots at most.

In connection with the Riemann hypothesis it appears to be important to find large classes of functions

$$
\sum_{-N}^{N} \alpha_{n} e^{n t} \quad\left(\alpha_{n}^{*}=\alpha_{-n}\right)
$$

with the property that the integral (1.9) has real roots only. For instance, this follows from Theorem, 11, if all the roots of (6.4) lie on the imaginary axis; but this result does not help much in the direction of the Riemann problem, which seems to be related to functions of the type

$$
\int_{-\infty}^{\infty} C(t) \prod_{n=1}^{N}\left(\mu_{n}^{2}+\cosh ^{2} t\right) e^{i z t} d t
$$

where $\mu_{1}, \cdots, \mu_{n}$ are real and $\geq 1$. In the following we establish some new results, which do not help either, but which may serve as material for observation.

Theorem 22. If $\lambda>0, \mu \geq 0$, the integrals $\Psi_{1}(z)=\int_{-\infty}^{\infty} C(t)(\mu+\cosh t) e^{i z t}$ $d t$ and $\Psi_{2}(z)=\int_{-\infty}^{\infty} C(t)\left(\mu+\cosh ^{2} t\right) e^{i z t} d t$ have real roots only.

Proof. (The reality of the roots of $\Psi_{1}(z)$ can also be proved as follows. It follows from Theorem 21 that $\Psi_{1}(z)$ has at most one root in the upper halfplane. Since $\Psi_{1}(z)$ is real and even, this possible one must be purely imaginary. But since the integrand is positive for $z$ purely imaginary such a root does not exist. The same argument is used in the proof of Theorem 23.) By partial integration we easily express $\Psi_{1}$ and $\Psi_{2}$ in terms of the functions $\Phi_{1}(z)$ and $\Phi_{2}(z)$ defined by (6.2):

$$
\begin{gathered}
z \Psi_{1}(z)=z \Phi_{1}(z)-\lambda \mu \Phi_{2}(z), \\
\lambda z \Psi_{2}(z)=z \Phi_{1}(z)+\left\{z^{2}-\lambda^{2}(1+\mu)\right\} \Phi_{2}(z) .
\end{gathered}
$$

The roots of the polynomials $z-i \lambda \mu$ and $z+i\left\{z^{2}-\lambda^{2}(1+\mu)\right\}$ lie in the upper half-plane and hence, by the lemma, (6.6) and (6.7) have real roots only (see the proof of Theorem 21). We notice that the same can be said if $-1 \leq$ $\mu \leq 0$, for then $\mu+\cosh t$ and $\mu+\cosh ^{2} t$ are universal factors.

On the other hand it is not difficult to show that both integrals have a pair of purely imaginary roots if $-\mu$ is positive and sufficiently large.

The following theorem is obtained by a generalization of the method employed above. The result, however, seems to be too complicated for application to any wide class of polynomials.

TheOREM 23. Let $\lambda$ be $>0$ and let $f(y)$ be a real polynomial, such that $\Phi^{\sim}(z)=$ $\int_{-\infty}^{\infty} C(t) f(\cosh t) e^{i z t} d t$ has real roots only. Then the same holds for the polynomial 
$f_{1}(y)=\mu y f(y)+f(y)+\lambda^{-1} f^{\prime}(y)+\lambda^{-2} f^{\prime \prime}(y)+\cdots$ if $\mu \geq 0$. It is also true for $\mu<0$, provided that $f_{1}(y)$ does not change sign for $y \geq 1$.

Proof. Putting

$$
\begin{aligned}
& \Phi_{1}^{\sim}(z)=\int_{-\infty}^{\infty}(C(t) f(\cosh t) \cosh t) e^{i z t} d t \\
& \Phi_{2}^{\sim}(z)=\int_{-\infty}^{\infty}(C(t) f(\cosh t) i \sinh t) e^{i z t} d t
\end{aligned}
$$

we find by partial integration

$$
z \int_{-\infty}^{\infty} C(t) f_{1}(\cosh t) e^{i z t} d t=\mu z \Phi_{1}^{\sim}(z)-\lambda \Phi_{2}^{\sim}(z) .
$$

Furthermore, we notice that the roots of $\Phi^{\sim}(z-i)=\Phi_{1}^{\sim}(z)-i \Phi_{2}^{\sim}(z)$ all have imaginary part 1 . For $\mu \geq 0$ the function $A(z)+i B(z)=\mu z-i \lambda$ has no roots in the lower half-plane so that the argument used in the proof of the preceding theorem shows that the function (6.8) has real roots only.

If $\mu<0$ we infer that (6.8) has one pair of conjugate complex roots at most. Since that function is an odd function of $z$, these possible roots must be purely imaginary. But if $f_{1}(y)$ does not change sign for $y \geq 1$ and if $z$ is purely imaginary, then $C(t) f_{1}(\cosh t) e^{i z t}$ does not change sign for $-\infty<t<\infty$. It follows that the integral does not vanish.

The following application may be of some interest. The function $f(y)=y^{N}$ satisfies the conditions of Theorem 23, for (6.1) has real roots only and $y^{N}=$ $(\cosh t)^{N}$ is a universal factor. Consequently Theorem 23 shows that, for $\lambda>0$,

$$
\int_{-\infty}^{\infty} C(t)\left\{1+\lambda \cosh t+\frac{\lambda^{2} \cosh ^{2} t}{2 !}+\cdots+\frac{\lambda^{N} \cosh ^{N} t}{N !}\right\} e^{i z t} d t
$$

has real roots only.

TheOREM 24. Let the polynomial $f(y)$ of degree $N$ have negative roots only, and let $\lambda$ be a number $\geq \frac{1}{2} N$. Then the function $\Phi^{\sim}(z)$ has real roots only.

It may be surmised that the condition $\lambda \geq \frac{1}{2} N$ can be replaced by a much weaker one.

Proof. We may assume that $f(y)$ has no roots for $-1 \leq y \leq 1$, the factors $y-a=\cosh t-a(-1 \leq a \leq 1)$ being universal factors (Theorem 11). We now proceed by double induction, in the first place with respect to the degree $N$ of $f(y)$ (the theorem is true for $N=0$ ) and secondly with respect to a number $n$, the smallest positive integer with the property that $f(y)$ has at least one root exceeding $-1-n / \lambda$. We shall reduce the case $(N, n)$ either to lower $N$ or to lower $n$; the case $(N, 0)$ will always be reduced to lower $N$.

Suppose that $f(y)$ (of degree $N$ ) has negative roots $<-1$ only, the largest of which, $\rho$ say, satisfies $-1-n / \lambda<\rho \leq-1-(n-1) / \lambda, n \geq 1$. Now 
consider the polynomial $f_{1}(y)=f^{\prime}(y)-\lambda f(y)$. Since $f(y)$ has real roots only, the same applies to $f_{1}(y)$. Now $f_{1}(y)$ has no roots for $y>1$ and exactly one root $\rho_{1}$ satisfying $\rho+\lambda^{-1}<\rho_{1} \leq 1$. Namely, $f^{\prime}(y) / f(y)$ decreases monotonically for $y \geq \rho$. For $y=\rho+\lambda^{-1}$ we have $f^{\prime}(y) / f(y)>(y-\rho)^{-1}=\lambda$, and, since the roots of $f(y)$ are supposed to be $<-1$, the inequality $f^{\prime}(1) / f(1)<\frac{1}{2} N \leq \lambda$ holds.

Now if $\rho_{1}<-1$, the polynomial $f_{1}(y)$ belongs to a case of lower $n$ and hence

$$
\int_{-\infty}^{\infty} C(t) f_{1}(\cosh t) e^{i z t} d t
$$

is supposed to have real roots only. If, however, $-1 \leq \rho_{1} \leq 1$, the polynomial $f_{2}(y)=f_{1}(y) /\left(y-\rho_{1}\right)$ is of degree $N-1$, and it follows that the roots of (6.10), with $f_{2}$ instead of $f_{1}$, are real. But since in that case cosh $t-\rho_{1}$ is a universal factor the same applies to (6.10) itself.

Again applying a universal factor, we find that

$$
i \int_{-\infty}^{\infty} C(t) f_{1}(\cosh t) \sinh t e^{i z t} d t=z \int_{-\infty}^{\infty} C(t) f(\cosh t) e^{i z t} d t
$$

has real roots only. This completes our induction.

We have not yet been able to generalize Theorem 24 to cases where $f(\cosh t)$ is an infinite product of factors $1+c_{\mu} \cosh t, c_{\mu}>0$. Such an extension might be given perhaps by carrying out a suitable reduction process and using Theorem 25 below. In Theorem 26 we shall use such a method in a different case, where a reduction process can be found indeed. Theorem 26 may be of some interest since it gives a result of a type we should like to have for functions of the form (6.5).

Theorem 25. If $\lambda>0,0<\delta<\frac{1}{2} \pi$, and if $n$ is a natural number, then there exists a positive number $\Delta(\lambda, \delta, n)$ with the property that the roots of

$$
\Psi(z)=\int_{-\infty}^{\infty} C(t) f(\cosh t) e^{i z t} d t
$$

lie in the strip $|\operatorname{Im} z| \leq \Delta(\lambda, \delta, n)$ for any real polynomial $f(y)$ of degree $n$ whose roots lie in the sector $\frac{1}{2} \pi+\delta \leq \arg y \leq \frac{3}{2} \pi-\delta$.

(It is possible to prove the same for the region consisting of the real axis and a circle $|z| \leq \Delta(\lambda, \delta, n)$. This can be done by introducing a denominator $\left(e^{t}+2+e^{-t}\right)$ (see the proof of Theorem 2).)

Proof. The function (6.1) can be developed as follows:

$$
\Phi(z)=\sum_{\nu=0}^{\infty} \frac{(-1)^{\nu}}{\nu !} \frac{\Gamma(i z-\nu)}{\left(\frac{1}{2} \lambda\right)^{i z-2 \nu}}+\sum_{\nu=0}^{\infty} \frac{(-1)^{\nu}}{\nu !} \frac{\Gamma(-i z-\nu)}{\left(\frac{1}{2} \lambda\right)^{i z-2 \nu}}
$$

(see (5.7) or [6]). It follows that for $\operatorname{Im} z<-1$ we have

$$
\left|\Phi(z)\left(\frac{1}{2} \lambda\right)^{i z} / \Gamma(i z)-1\right|<|z|^{-1} A(\lambda)
$$


$A=A(\lambda)$ independent of $z$. Consequently, the function

$$
\Phi_{k}(z)=\int_{-\infty}^{\infty} C(t)(\cosh t)^{k} e^{i z t} d t=2^{-k} \sum_{\mu=0}^{k}\left(\begin{array}{l}
k \\
\mu
\end{array}\right) \Phi\{z+(k-2 \mu) i\}
$$

satisfies

$$
\left|\Phi_{k}(z) \lambda^{i z+k} 2^{-i z} / \Gamma(i z)(i z)^{k}-1\right|<A(\lambda, k) /|z| \quad(\operatorname{Im} z<-1) .
$$

Let $A_{0}(\lambda, n)$ be the largest of the numbers $A(\lambda), A(\lambda, 1), \cdots, A(\lambda, n)$, and let $f(y)=\left(1+c_{1} y\right) \cdots\left(1+c_{n} y\right)=a_{0}+a_{1} y+\cdots+a_{n} y^{n}$, where $\left|\arg c_{\mu}\right|<$ $\frac{1}{2} \pi-\delta, a_{\mu}$ real. Then we have $\Psi(z)=a_{0} \Phi_{0}(z)+a_{1} \Phi_{1}(z)+\cdots+a_{n} \Phi_{n}(z)$, and by (6.12), for $\operatorname{Im} z<-1$,

$$
\left|\Psi(z)\left(\frac{1}{2} \lambda\right)^{i z} / \Gamma(i z)-\sum_{k=0}^{n} a_{k}(i z / \lambda)^{k}\right|<A_{0}(\lambda, n)|z|^{-1} \sum_{k=0}^{n}\left|a_{k} z^{k}\right| \lambda^{-k} .
$$

Consequently

$$
\left|\frac{\Psi(z)\left(\frac{1}{2} \lambda\right)^{i z}}{\Gamma(i z)}-\prod_{\mu=1}^{n}\left(1+\frac{c_{\mu} i z}{\lambda}\right)\right|<\frac{A_{0}(\lambda, n)}{|z|} \prod_{\mu=1}^{n}\left(1+\left|\frac{c_{\mu} z}{\lambda}\right|\right) .
$$

Here $c_{\mu} i z / \lambda=d_{\mu}$ satisfies $\left|\arg d_{\mu}\right| \leq \pi-\delta$ and so $\left|1+d_{\mu}\right| \geq \sin \frac{1}{2} \delta\left(1+\left|d_{\mu}\right|\right)$ is easily verified. It now follows from (6.13) that $\Psi(z) \neq 0$ if $z$ satisfies $\operatorname{Im} z$ $<-1$ and $|z|>A_{0}(\lambda, n)\left(\sin \frac{1}{2} \delta\right)^{-n}$. Since $\Psi(z)$ is real for real values of $z$, an analogous result holds for $z$ in the upper half-plane, so that $\Delta(\lambda, \delta, n)=\operatorname{Max}$ $\left\{A_{0}(\lambda, n)\left(\sin \frac{1}{2} \delta\right)^{-n}, 1\right\}$ has the required property.

Theorem 26. Suppose that $\lambda>0$ and that the roots of the real polynomial $f(y)$ all lie on the negative real axis. If $f^{\prime}(1) / f(1) \leq 2 \lambda$, then the function

$$
\Psi(z)=\int_{-\infty}^{\infty} C_{2}(t) f(\cosh t) e^{i z t} d t
$$

where $C_{2}(t)=\exp \left(-\lambda \cosh ^{2} t\right)$, has real roots only.

Proof. We use the following Lemma which is not actually a special case of Theorem 25 but which can be proved in the same way without any essential difficulty. (First transform $t=\frac{1}{2} \tau$.) In fact it is an easier case; we omit its proof.

Lemma 3. If $\lambda>0$ and $n$ a natural number, then there exists a positive number $\Delta(\lambda, n)$, such that the roots of (6.14) satisfy $|\operatorname{Im} z| \leq \Delta(\lambda, n)$ for any polynomial $f(y)$ of degree $n$ with negative roots only.

For given values of $\lambda$ and $n$, let $\Delta_{0}(\lambda, n)$ be the smallest possible of the numbers $\Delta$ with the following property: The roots of (6.14) lie in the strip $|\operatorname{Im} z| \leq \Delta$ for any polynomial $f(y)$, of degree $\leq n$, satisfying the conditions of our theorem. Lemma 3 shows that such a number $\Delta_{0}(\lambda, n)$ exists. We shall assume $\Delta_{0}(\lambda, n)>$ 0 , and show that to be contradictory. 
Let the polynomial $f(y)$ of degree $n$ satisfy the conditions of our theorem. By partial integration we find

$$
z \Psi(z)=\int_{-\infty}^{\infty}\left(C_{2}(t) f_{1}(\cosh t) i \sinh t\right) e^{i z t} d t,
$$

where $f_{1}(y)=f^{\prime}(y)-2 \lambda y f(y)$.

Since $f^{\prime}(1) / f(1) \leq 2 \lambda, f_{1}(y)$ has exactly one root, say $a$, in the interval $0 \leq y \leq 1$. Now the polynomial $f_{2}(y)=f_{1}(y) /(y-a)$ has $n$ negative roots $\rho_{1}, \rho_{2}, \cdots, \rho_{n}$, satisfying

$$
\rho_{1} \leq \sigma_{1} \leq \rho_{2} \leq \sigma_{2} \leq \cdots \leq \rho_{n} \leq \sigma_{n}<0,
$$

where $\sigma_{1}, \cdots, \sigma_{n}$ denote the roots of $f(y)$. This is easily seen by drawing graphs of $f^{\prime}(y) / f(y)$ and of $2 \lambda y$. It follows from (6.16) that $f_{2}^{\prime}(1) / f_{2}(1) \leq f^{\prime}(1) / f(1)$, so that $f_{2}(y)$ also satisfies the conditions of Theorem 26 . Hence the roots of $\int_{-\infty}^{\infty} C_{2}(t) f_{2}(t) e^{i z t} d t$ lie in the strip $|\operatorname{Im} z| \leq \Delta_{0}(\lambda, n)$.

Since $i \sinh t$ and (cosh $t-a$ ) are strong universal factors the roots of (6.15) lie in a narrower strip $|\operatorname{Im} z| \leq \Delta_{1}(\lambda, n), \Delta_{1}(\lambda, n)<\Delta_{0}(\lambda, n)$. Namely, by virtue of Theorem 11 we may take $\Delta_{1}(\lambda, n)=\left\{\Delta_{0}^{2}(\lambda, n)-1\right\}^{\frac{1}{2}}$ if $\Delta_{0}>1$ and $\Delta_{1}(\lambda, n)=0$ if $\Delta_{0}<1$.

As this holds for any polynomial $f(y)$ satisfying the conditions of our theorem it contradicts the minimum property of $\Delta_{0}(\lambda, n)$. It follows that $\Delta_{0}(\lambda, n)=0$, which proves our theorem.

The condition $f^{\prime}(1) / f(1) \leq 2 \lambda$ in Theorem 26 is a natural one. It is equivalent to the condition that the function $C_{2}(t) f(\cosh t)$, occurring in the integrand of (6.14), has its maximum for $t=0$ and decreases steadily for $t>0$ (it is, of course, a real and even function of $t$ ).

Theorem 26 can be extended to infinite products immediately.

Theorem 27. If $c_{1}, c_{2}, \cdots$ are positive numbers and if $\sum_{\mu=1}^{\infty} c_{\mu}\left(1+c_{\mu}\right)^{-1} \leq 2 \lambda$, then the function $\int_{-\infty}^{\infty} C_{2}(t) \prod_{\mu-1}^{\infty}\left(1+c_{\mu} \cosh t\right) e^{i z t} d t$ has real roots only.

7. Remarks concerning the Riemann hypothesis. The special interest devoted to integrals of the type (1.9) arose from the Riemann hypothesis concerning the $\zeta$-function. Putting $s=\frac{1}{2}+i z$ and writing (see [12; Chapter 3])

$$
\begin{gathered}
\Xi(z)=\xi(s)=\frac{1}{2} s(s-1) \Gamma\left(\frac{1}{2} s\right) \pi^{-\frac{1}{2} s} \zeta(s), \\
\varphi(t)=\sum_{n=1}^{\infty}\left(2 n^{4} \pi^{2} e^{9 t / 4}-3 n^{2} \pi e^{5 t / 4}\right) e^{-n^{2} \pi e^{t}},
\end{gathered}
$$

we have

$$
\Xi(2 z)=\int_{-\infty}^{\infty} \varphi(t) e^{i z t} d t
$$

here $\varphi(t)$ is an even function of $t$. Riemann conjectured that (7.3) has real roots only. 
It is known from Euler's product expansion $\zeta(s)=\prod_{p}\left(1-p^{-s}\right)^{-1}$ and from the functional equation $\Xi(z)=\Xi(-z)$ that the roots of (7.3) lie in the strip $|\operatorname{Im} 2 z| \leq \frac{1}{2}$ anyhow. Hence it follows, by Theorem 12, that

$$
\int_{-\infty}^{\infty} \varphi(t) \cosh \frac{1}{4} t e^{i z t} d t
$$

has real roots only. (See [9].)

It would be very interesting to have a proof for the reality of the roots of (7.4) which does not use the known fact that $\zeta(s) \neq 0$ for $\operatorname{Re} s>1$, but only employs the properties of $\varphi(t) \cosh \frac{1}{4} t$ and the general properties of trigometric integrals. Such a proof might open the way to the Riemann hypothesis.

Neither Pólya's work nor the present paper gave any direct information concerning these problems thus far. But there are some interesting facts. Pólya [8] noted that, if $t \rightarrow \pm \infty$,

$$
\varphi(t) \sim \varphi_{1}(t)=4 \pi^{2}(\cosh 9 t / 4) \exp (-2 \pi \cosh t)
$$

where $\int_{-\infty}^{\infty} \varphi_{1}(t) e^{i z t} d t$ has real roots only, since $\cosh 9 t / 4$ is a universal factor.

A closer approximation to $\varphi(t)$ is

$$
\begin{gathered}
\varphi(t)=\varphi_{2}(t)+O\left(e^{t / 4} \exp (-2 \pi \cosh t)\right) \\
\varphi_{2}(t)=\left\{4 \pi^{2} \cosh \frac{9 t}{4}+\left(4 \pi^{3}-6 \pi\right) \cosh \frac{5 t}{4}+4 \pi^{2} \cosh \frac{t}{4}\right\} \exp (-2 \pi \cosh t),
\end{gathered}
$$

and again $\int_{-\infty}^{\infty} \varphi_{2}(t) e^{i z t} d t$ has real roots only. (Pólya gives a second approximation with $\left\{16 \pi^{2} \cosh 9 t / 4-24 \pi \cosh 5 t / 4\right\}$ (see [8], [12; 45]). This approximation however is not actually closer than $\varphi_{1}(t)$.) We have, namely,

$$
\varphi_{2}(t)=2(\cosh 5 t / 4)\left\{2 \pi^{3}-3 \pi+4 \pi^{2} \cosh t\right\} \exp (-2 \pi \cosh t),
$$

so that application of Theorem 22 and of the universal factor cosh $5 t / 4$ gives the result.

We must not expect that the function $\Xi(t)$ can be approximated by functions $\int_{-\infty}^{\infty}(\exp (-2 \pi \cosh t)) f_{n}(t) e^{i z t} d t$ where the $f_{n}$ are analytic universal factors. For if $f_{n}(t) \rightarrow f(t)$ in a circle $|t|<\epsilon$, then $f(t)$ can be continued over the whole plane. (See [4].) This is not true for the function $\varphi(t)$, which cannot be continued over the lines $\operatorname{Im} t= \pm \frac{1}{2} \pi$. The results of the preceding section however suggest approximations of a different type.

In the following, we shall devote our attention to Ramanujan's function instead of the Riemann function. Owing to the pole at $s=1$, the formulas for Riemann's function show a complication which is probably unessential.

Let $\tau(n)$ be the coefficients of the power series for the well-known elliptic modular function,

$$
g(y)=y\left\{(1-y)\left(1-y^{2}\right) \cdots\right\}^{24}=\sum_{1}^{\infty} \tau(n) y^{n} .
$$


With these coefficients Ramanujan [2; Lecture $\mathrm{X}$ ] constructed his zeta-function $F(s)=\sum_{1}^{\infty} \tau(n) / n^{s}=\prod_{v}\left\{1-\tau(p) p^{-s}+p^{11-2 s}\right\}^{-1}$. The series and the product are absolutely convergent and $\neq 0$ for $\operatorname{Re} s>13 / 2$. $F(s)$ can be continued over the whole plane by the formula

$$
\Xi^{\sim}(i s)=(2 \pi)^{-8-6} F(s+6) \Gamma(s+6)=\int_{0}^{\infty} x^{s+5} g\left(e^{-2 \pi x}\right) d x .
$$

It follows from $x^{6} g\left(e^{-2 \pi x}\right)=x^{-6} g\left(e^{-2 \pi / x}\right)$ that $\Xi^{\sim}(z)$ satisfies the functional equation $\Xi^{\sim}(z)=\Xi^{\sim}(-z)$. By virtue of the above functional equation of $g(z)$ we have

$$
\begin{aligned}
x^{6} g\left(e^{-2 \pi x}\right) & =\left\{g\left(e^{-2 \pi x}\right) g\left(e^{-2 \pi / x}\right)\right\}^{\frac{1}{2}} \\
& =e^{-\pi\left(x+x^{-1}\right)} \prod_{\nu=1}^{\infty}\left\{\left(1-e^{-2 \pi \nu x}\right)\left(1-e^{-2 \pi \nu / x}\right)\right\}^{12} .
\end{aligned}
$$

It follows from (7.7) that

$$
\Xi^{\sim}(z)=\int_{-\infty}^{\infty} \varphi^{\sim}(t) e^{i z t} d t
$$

where

$$
\varphi^{\sim}(t)=(\exp (-2 \pi \cosh t)) \prod_{\nu=1}^{\infty}\left\{\left(1-e^{-2 \pi \nu e^{t}}\right)\left(1-e^{-2 \pi \nu e^{-t}}\right)\right\}^{12} .
$$

In analogy with the Riemann hypothesis we have the problem as to the reality of all the roots of (7.9). Anyhow, the roots lie in the strip $|\operatorname{Im} z| \leq \frac{1}{2}$. We prefer the discussion of (7.9) to that of (7.3) since $\varphi^{\sim}(t)$ has a simple product expansion.

There are several aspects connecting the problem concerning the roots of (7.9) with the results and methods of the preceding sections.

(a). A first indication is given by the domain of regularity and the behaviour for $t \rightarrow \pm \infty$ of $\varphi^{\sim}(t)$, in connection with Theorems 2 and 25 . We have namely, introducing a function $q^{\sim}(x)$,

$$
\tilde{\varphi} \sim(t)=(\exp (-4 \pi \cosh t)) q^{\sim}(x) \quad\left(x=e^{t}\right)
$$

where $q^{\sim}(x)$ is regular for $\operatorname{Re} x>0$, but cannot be continued over the imaginary axis. Furthermore $q^{\sim}(x)=q^{\sim}\left(1 / x^{*}\right)$ and $q^{\sim}(x) \sim x^{6}$ for $\operatorname{Re} x \rightarrow+\infty$, so that $q^{\sim}(x)$ is relatively smooth for $x \rightarrow \infty$ and $x \rightarrow 0$. The nature of $q^{\sim}(x)$ is therefore related to that of the functions $q(x)$ occurring in Theorem 2 (for the special case $P(t)=4 \pi \cosh t$ ), which lead to trigonometric integrals with a finite number of non-real roots at most. These $q(x)$ had to be regular in a sector $|\arg x| \leq$ $\frac{1}{2} \pi+\delta, \delta>0$, and meromorphic at $x=0$ and $x=\infty$.

It turns out to be important to construct large classes of functions $q(x)$ for which the finite number of non-real roots is either zero or uniformly bounded. No such functions have been found yet, apart from polynomials $q(x)$ (see $\$ 6$ ). 
(b). The infinite product in (7.10) reminds of the functions in Theorems 24-27. Consider

$$
\Xi_{N}^{\sim}(z)=\int_{-\infty}^{\infty}(\exp (-2 \pi \cosh t)) \prod_{\nu=1}^{N}\left\{\left(1-e^{-2 \pi \nu \theta^{t}}\right)\left(1-e^{-2 \pi \nu e^{-t}}\right)\right\}^{12} e^{i z t} d t
$$

We have

$$
\begin{aligned}
\left(1-e^{-2 \pi \nu e^{t}}\right)(1- & \left.e^{-2 \pi \nu e^{-t}}\right) \\
& =(\exp (-2 \pi \nu \cosh t)) 4 \pi^{2} \nu^{2} \prod_{k=1}^{\infty}\left\{\left(1+\nu^{2} k^{-2} e^{2 t}\right)\left(1+\nu^{2} k^{-2} e^{-2 t}\right)\right\} \\
& =4 \pi^{2} \nu^{2}(\exp (-2 \pi \nu \cosh t)) \prod_{k=1}^{\infty}\left\{\left(1-\nu^{2} k^{-2}\right)^{2}+4 \nu^{2} k^{-2} \cosh ^{2} t\right\} .
\end{aligned}
$$

Hence $\Xi_{N}^{\sim}(z)$ can be written in the form

$$
C \int_{-\infty}^{\infty} C(t) \prod_{\mu}\left(1+\mu^{-2} \cosh ^{2} t\right) e^{i z t} d t
$$

where $\mu$ runs through an infinite set of real numbers and $\lambda>0$. This form reminds of Theorem 27, although for functions of the type (7.13) we may not expect a result as general as that theorem. We have to face the rather disappointing fact that the functions $\Xi_{N}^{\sim}(z)$ may have an infinity of non-real roots (see (7.18) below). This does not mean that it is no use considering functions of the type (7.13), for $\Xi^{\sim}(z)$ can of course also be approximated directly by functions of the type (7.13) without using $\Xi_{N}^{\sim}(z)$.

(c). It may be expected that the reality of the roots of a trigonometric integral is in some way connected with multiplicative properties of the integrand. Several theorems in $\$ 6$ point in this direction. Therefore the fact that the integrand of (7.9) is given in the form of an infinite product may be important.

As an example of a multiplication theorem we give the following one which is, however, too weak to be of any use here.

Theorem 28. Let the function $\varphi_{1}(t)$ and $\varphi_{2}(t)$ be continuous for $-\infty<t<\infty$ and $O\left(e^{-t^{2}}\right)$ for some positive $\epsilon,\left(\varphi_{1}(t)\right)^{*}=\varphi_{1}(-t),\left(\varphi_{2}(t)\right)^{*}=\varphi_{2}(-t)$. Suppose that $\varphi_{1}$ and $\varphi_{2}$ are such that the integrals $\int_{-\infty}^{\infty} e^{-\lambda t^{2}} \varphi_{i}(t) e^{i z t} d t, j=1,2$, have real roots only, whatever $\lambda, \lambda \geq 0$, may be. Then the same property holds for the product $\varphi_{1}(t) \varphi_{2}(t)$.

Proof. For $\lambda \geq 0$, the product

$$
\begin{aligned}
& \int_{-\infty}^{\infty} e^{-\lambda t^{2}} \varphi_{1}(t) e^{i z t} d t \int_{-\infty}^{\infty} e^{-\lambda s^{2}} \varphi_{2}(s) e^{i z s} d s \\
& \quad=\frac{1}{2} \int_{-\infty}^{\infty} e^{i z \tau} d \tau \int_{-\infty}^{\infty} e^{-\lambda\left(\sigma^{2}+\tau^{2}\right)} \varphi_{1}\left(\frac{1}{2}(\tau-\sigma)\right) \varphi_{2}\left(\frac{1}{2}(\tau+\sigma)\right) d \sigma
\end{aligned}
$$


has real roots only. To the right side we apply the universal factor $e^{\text {in } \tau^{2}}$ and find that also $\int_{-\infty}^{\infty} e^{i z \tau} d \tau \int_{-\infty}^{\infty} e^{-\frac{1}{2 \lambda} \sigma^{2}} \varphi_{1}\left(\frac{1}{2}(\tau-\sigma)\right) \varphi_{2}\left(\frac{1}{2}(\tau+\sigma)\right) d \sigma$ has real roots only. Now making $\lambda \rightarrow \infty$, we easily deduce that $\int_{-\infty}^{\infty} \varphi_{1}(t) \varphi_{2}(t) e^{i z t} d t$ has real roots only. On applying this to $e^{-\lambda t^{2}} \varphi_{1}(t)$ instead of $\varphi_{1}(t)$ the same follows concerning $\int_{-\infty}^{\infty} e^{-\lambda t^{2}} \varphi_{1}(t) \varphi_{2}(t) e^{i z t} d t$.

Functions $\varphi(t)$ satisfying the conditions of the theorem are, for instance,

$$
\varphi(t)=\exp \left(-a t^{4}-i b t^{3}-c t^{2}-i d t\right)
$$

where $a, b, c, d$ are real and either $a>0$ or $a=0, b \neq 0$ or $a=b=0, c>0$. Namely, it follows from Theorem 1 that $\int_{-\infty}^{\infty} e^{-a t^{2}-c t^{2}} e^{i z t} d t, a>0, c \geq 0$, has real roots only; since $e^{p t^{2}}, p>0$, is a universal factor we may drop the restriction about $c$. The statement concerning (7.14) can now be proved on using a substitution $t=t^{\sim}+i q$; the cases with $a=0$ can be tackled by a limit process.

No items essentially different from (7.14) have been found yet; thus Theorem 28 gave no new trigonometric integrals with real roots only.

(d). Functions of the type (7.12) can be discussed directly by the method developed in $\$ 5$. In a simple case we shall find an infinity of non-real roots. The general case is difficult to deal with but must be expected to show the same effect.

We consider the function

$$
f(z)=\int_{-\infty}^{\infty}(\exp (-2 \pi \cosh t))\left(\sum_{\nu=0}^{n} \gamma_{\nu} e^{-2 \pi \nu e^{t}}\right)\left(\sum_{\nu=0}^{n} \gamma_{\nu}^{*} e^{-2 \pi \nu e^{-t}}\right) e^{i z t} d t
$$

and suppose that for a certain integer $k \geq 0$ the polynomial $P(y)=\gamma_{0}+\gamma_{1} y+$ $\cdots+\gamma_{n} y^{n}$ satisfies

$$
P(1)=P^{\prime}(1)=P^{\prime \prime}(1)=\cdots=P^{(k-1)}(1)=0, P^{(k)}(1) \neq 0 .
$$

We also introduce the function

$$
\psi(s)=\pi^{-s}\left\{\gamma_{0}+\gamma_{1} 3^{-s}+\gamma_{2} 5^{-\cdot}+\cdots+\gamma_{n}(2 n+1)^{-s}\right\}
$$

We define the coefficients $\beta_{k}, \beta_{k+1}, \cdots$ by (see (5.3))

$$
e^{-\pi e^{-t}} \sum_{\nu=0}^{n} \gamma_{\nu}^{*} e^{-2 \pi v e^{-t}}=\sum_{\nu=k}^{\infty} \beta_{\nu} e^{-\nu t}
$$

the terms with $e^{-\nu t}, 0 \leq \nu \leq k$, vanish by virtue of (7.16). The following function plays the same role as $H(s)$ did in $\S 5$ :

$$
H^{\sim}(s)=\int_{0}^{\infty} e^{-\pi u} \sum_{0}^{n} \gamma_{\nu} e^{-2 \pi v u} u^{s} d u=\psi(s+1) \Gamma(s+1) .
$$


The process carried out in $\$ 5$ now leads to (see (5.7))

$$
f(z)=\sum_{k}^{\infty} \beta_{\nu} \psi(i z-\nu) \Gamma(i z-\nu)+\sum_{k}^{\infty} \beta_{\nu}^{*} \psi^{*}(-i z-\nu) \Gamma(-i z-\nu),
$$

from which some information on the roots of $f(z)$ can be obtained.

From (7.18) we can deduce that if all but a finite number of roots of (7.17) lie in the half-plane $\operatorname{Re} s \geq \omega$, then all but a finite number of roots of $f(z)$ lie in the strip $|\operatorname{Im} z| \leq \operatorname{Max}(k+\omega+\delta, \delta)$, for any $\delta>0$. Namely, if $\operatorname{Im} z<0$, the major contribution to (7.18) is given by $\beta_{k} \psi(i z-k) \Gamma(i z-k)$.

Now turning to the integrals (7.12) we see that $k$ is large, $k=12 N$, but it is not likely that the number $\omega$ introduced above is $<-k$. So probably the functions (7.12) have an infinity of non-real roots.

In a simple case, namely,

$$
f_{1}(z)=\int_{-\infty}^{\infty}(\exp (-2 a \cosh t))\left(1-e^{-b e^{t}}\right)\left(1-e^{-b e^{-t}}\right) e^{i z t} d t
$$

where $a>0, b>0$, we show that an infinity of non-real roots exist. We have $\psi_{1}(s)=a^{-s}-(a+b)^{-s}$, whose roots are $s=2 v \pi i / \log (1+b / a), \nu=0, \pm 1$, $\pm 2, \cdots$. It follows from (7.18) that for $|\operatorname{Re} z|>1, \operatorname{Im} z<-\delta, 0<\delta<\frac{1}{2}$, we have $f_{1}(z)=b \psi_{1}(i z-1) \Gamma(i z-1)+O\left\{z^{-2 \delta} \Gamma(i z-1)\right\}$. Now, by Rouché's theorem, we infer that $f_{1}(z)$ has, from a certain value of $|\nu|$ onward, just one root in the neighborhood of any point $-i+2 \nu \pi / \log (1+b / a)$. Apart from these $f_{1}(z)$ has but a finite number of roots for $\operatorname{Im} z<-\delta$.

This failure must not be considered as an argument against the Riemann hypothesis. Objections of the same "strength" could be used against the true fact that the roots of $\int_{-\infty}^{\infty} \varphi^{\sim}(t) \cosh \frac{1}{2} t e^{i z t} d t$ (see (7.10) and (7.4)) are real.

I express my gratitude to Professor Pólya for his kind interest in the results, and to Messrs. J. Korevaar and F. van der Blij, of the Mathematisch Centrum, Amsterdam, for reading the manuscript and indicating many corrections.

\section{REFERENCES}

1. N. G. De Bruisn, On the zeros of a polynomial and of its derivative, I., Koninklijke Nederlandsche Akademie van Wetenschappen, Proceedings, vol. 49(1946), pp. 1037-1044 (= Indagationes Mathematicae ex Actis Quibus Titulis, Proceedings of the Section of Sciences, vol. 8(1946), pp. 635-642.

2. G. H. HARDy, Ramanujan; Twelve Lectures on Subjects Suggested by His Life and Work, Cambridge, England, 1940.

3. J. L. W. V. JENSEN, Recherches sur la theorie des équations, Acta Mathematica, vol. 36(1913), pp. 181-195.

4. G. PóLYA, Algebraische Untersuchungen über ganze Funktionen vom Geschlechte Null und Eins, Journal für die reine und angewandte Mathematik, vol. 145(1915), pp. 224-249.

5. Georg Pólya, Über die Nullstellen gewisser ganzen Funktionen, Mathematische Zeitschrift, vol. 2(1918), pp. 352-383.

6. G. P6LYA, Bemerkung über die Integraldarstellung der Riemannschen $\xi-F u n k t i o n$, Acta Mathematica, vol. 48(1926), pp. 305-317. 
7. G. Pólya, On the zeros of certain trigonometric integrals, Journal of the London Mathematical Society, vol. 1(1926), pp. 98-99.

8. G. Pólya, Über trigonometrische Integrale mit nur reellen Nullstellen, Journal für die reine und angewandte Mathematik, vol. 158(1927), pp. 6-18.

9. G. PóLya, Über die algebraisch-funktionentheoretischen Untersuchungen von $J . L . W . V$. Jensen, Det Kgl. Danske Videnskabernes selskab. Mathematisk-fisiske Meddelelser. Kobenhavn, vol. 7(1927), pp. 3-33.

10. G. Pólya and G. Szegö, Aufgaben und Lehrsätze aus der Analysis, vol. 1, Berlin, 1925.

11. G. Pólya And G. Szegö, Aufgaben und Lehrsätze aus der Analysis, vol. 2, New York, 1945.

12. E. C. Titchmarsh, The Zeta-Function of Riemann, Cambridge, England, 1930.

Mathematisch Institudt der Technische Hogeschool

Delft, Netherlands. 